\title{
A Recursive Decomposition Method for Large Scale Continuous Optimization
}

\author{
Yuan Sun, Michael Kirley, and Saman K. Halgamuge
}

\begin{abstract}
Cooperative Co-evolution (CC) is an evolutionary computation framework that can be used to solve high dimensional optimization problems via a 'divide-and-conquer' mechanism. However, the main challenge when using this framework lies in problem decomposition. That is, deciding how to allocate decision variables to a particular sub-problem, especially interacting decision variables. Existing decomposition methods are typically computationally expensive. In this paper, we propose a new decomposition method, which we call Recursive Differential Grouping (RDG), by considering the interaction between decision variables based on non-linearity detection. RDG recursively examines the interaction between a selected decision variable and the remaining variables, placing all interacting decision variables into the same sub-problem. We use analytical methods to show that RDG can be used to efficiently decompose a problem, without explicitly examining all pairwise variable interactions. We evaluated the efficacy of the RDG method using large scale benchmark optimization problems. Numerical simulation experiments showed that RDG greatly improved the efficiency of problem decomposition in terms of time complexity. Significantly, when RDG was embedded in a CC framework, the optimization results were better than results from seven other decomposition methods.
\end{abstract}

Index Terms-Large scale global optimization, cooperative coevolution, decomposition method, continuous optimization problem.

\section{INTRODUCTION}

$\mathbf{L}$ ARGE-SCALE (high-dimensional) optimization problems are ubiquitous in the real-world, occurring in domains spanning the sciences, engineering, and multidisciplinary design problems [1]-[3]. Such problems are very difficult to solve when using evolutionary algorithms (EAs), and in many cases cannot be solved when using traditional mathematical approaches. This in part may be attributed to the fact that (a) the search space of an optimization problem grows exponentially as the dimensionality increases [4]; (b) the complexity of an optimization problem usually grows as the dimensionality increases [5]; and (c) the computational cost of using some EAs (e.g., estimation of distribution algorithms) when solving very high-dimensional problems is extremely high [6].

Yuan Sun is with the Department of Mechanical Engineering, The University of Melbourne, Parkville, VIC, 3010, Australia, email: yuans2@student.unimelb.edu.au.

Michael Kirley is with the Department of Computing and Information Systems, The University of Melbourne, Parkville, VIC, 3010, Australia email: mkirley@unimelb.edu.au.

Saman K. Halgamuge is with the Research School of Engineering, The Australian National University, Canberra, ACT, 2601, Australia, email: saman.halgamuge@anu.edu.au.

Manuscript received August 15, 2016; revised November 30, 2017, March 20, 2017, and July 29, 2017; accepted November 20, 2017.
There has been significant recent interest within the Evolutionary Computation community focussed specifically on tackling large scale global optimization (LSGO) problems. This is best illustrated by the introduction of special sessions held at the leading conferences and the special issues published in related journals. The review papers by Mahdavi et al., [7] and LaTorre et al., [8] highlight recent developments in this exciting field.

Cooperative Co-evolution (CC) [9] has been used with some success when 'scaling up' EAs to tackle very high dimensional search and optimization problems. For example, CC has been applied to large scale continuous [10], combinatorial [11], constrained [12], multi-objective [13] and dynamic [14] optimization problems. The CC framework divides the LSGO problem into a number of sub-components, and uses an (several) EA(s) to solve each sub-component cooperatively. When optimizing each sub-component, representatives (typically the best sub-solutions found) from the other sub-components are combined with individuals in the optimized sub-component, to form complete candidate solutions that can be evaluated. A number of studies have shown that the problem decomposition can have a significant impact on the performance of a CC framework (e.g., [10], [15]-[18]).

The existing decomposition methods can be classified into two very different approaches (see Section II-B for a brief review): In the Manual Decomposition method, the structure of the sub-components is manually designed (e.g., Random Grouping [19]). This method does not take the underlying structure of variable interactions (see Section II-B1 for formal definition) into consideration. In the second method, Automatic Decomposition, the structure of the sub-components is determined by the identified decision variable interactions (e.g., Differential Grouping [10]). However, this approach can be computationally expensive - decomposing an $n$-dimensional problem typically consumes $\mathcal{O}\left(n^{2}\right)$ function evaluations (FEs). This high computational complexity results in an inappropriate allocation of computational resources to the decomposition stage rather than the optimization stage.

In this paper, we propose a Recursive Differential Grouping (RDG) method, which can decompose an $n$-dimensional problem using $\mathcal{O}(n \log (n))$ FEs. The RDG method examines the interaction between a selected decision variable $x_{i}$ and the remaining decision variables based on non-linearity detection. If any interaction is identified, the remaining decision variables will be divided into two equally sized groups, and the interaction between $x_{i}$ and each group is checked. This process is carried out recursively until all of the individual decision variables interacting with $x_{i}$ have been identified and 
have been placed into the same sub-component as $x_{i}$.

We have evaluated the efficacy of the RDG method using benchmark LSGO problems (problems from the special sessions on LSGO at CEC'2010 [20] and CEC'2013 [21]). Comprehensive numerical simulations showed that the RDG method can decompose the benchmark problems efficiently in terms of time complexity. We verified that the sub-component groupings dictated by RDG were in fact useful for optimization. When embedded into a CC framework, the optimization results generated using the RDG method were statistically better in terms of solution quality when compared against seven other decomposition methods across the benchmark suite.

The remainder of this paper is organized as follows. Section II describes the state-of-the-art algorithms and decomposition methods within the context of LSGO. Section III describes the proposed RDG method in detail. Section IV describes experiments to evaluate the proposed RDG method. Section $\mathrm{V}$ presents and analyzes the experimental results. Section VI concludes the paper and shows future directions.

\section{RELATED WORK}

\section{A. Algorithms for $L S G O$}

In this section, we briefly describe the state-of-the-art techniques that can be used to 'scale up' EAs for LSGO problems.

1) Cooperative Co-evolution: The CC [9] framework tackles a LSGO problem using a divide-and-conquer strategy. It divides the problem into a number of low-dimensional subcomponents that are solved cooperatively. A standard CC algorithm consists of two stages: decomposition and optimization.

In the decomposition stage, an optimization problem is decomposed into several sub-components. For example, a decomposition of a 6 -dimensional optimization problem $(f$ : $\left.\mathbb{R}^{6} \rightarrow \overline{\mathbb{R}}\right)$ could possibly be $\left\{\left(x_{1}, x_{2}\right),\left(x_{3}, x_{4}\right),\left(x_{5}, x_{6}\right)\right\}$, as shown in Fig. 1. When the structure of the underlying decision variable interactions are considered, this allocation to subcomponents may in fact be different. Recent studies have shown that the performance of a CC algorithm relies heavily on the way the optimization problem is decomposed [7], [10], [15], [16], [18]. See sections II-B and III for further details.

In the optimization stage, an evolutionary algorithm can be used to optimize each sub-component based on a context vector. The context vector is a complete candidate solution, typically consisting of the best sub-solutions from each subcomponent. When optimizing the $i_{t h}$ sub-component, the context vector (excluding the $i_{t h}$ sub-solution) is used to combine with the individuals in the $i_{t h}$ sub-component, to form complete candidate solutions that can be evaluated, as shown in Fig. 1. It has been found that using only one context vector may be too greedy [22]. Therefore, the adaptive multi-context $\mathrm{CC}$ [22] framework is proposed, which employs more than one context vector to co-evolve sub-components. The original CC framework [9] optimizes each sub-component in a round-robin fashion. Recently, a contribution based CC framework [23] has been proposed to efficiently allocate the computational resources. In each cycle, it selects and optimizes the subcomponent that makes the greatest historical contribution to the fitness improvement.

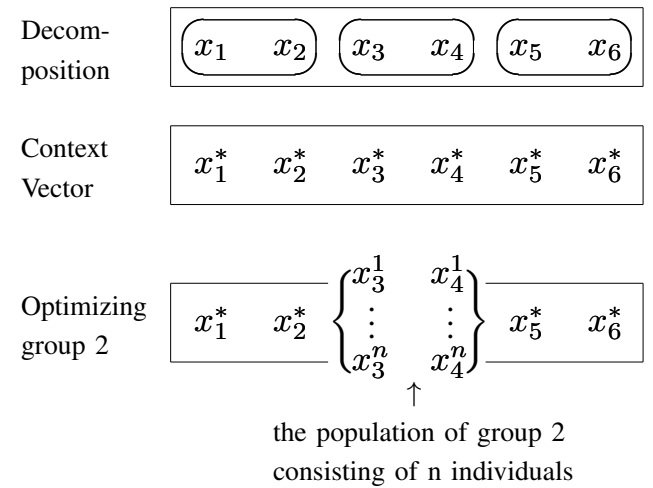

Fig. 1. The decomposition and optimization of a 6-dimensional problem using a CC algorithm. The problem has been decomposed into 3 sub-components, each with 2 decision variables. When optimizing the $2_{n d}$ sub-component, the context vector (excluding the $2_{n d}$ sub-solution) is used to combine with the individuals in the $2_{n d}$ sub-component, to form complete candidate solutions that can be evaluated.

2) Other Techniques: In addition to $\mathrm{CC}$, there are other techniques that can be used to address the additional challenges inherent in LSGO problems. Representative techniques include the model complexity control [6] and random projection [24] methods for estimation of distribution algorithms; the multiple strategies [25] and generalized opposition-based learning [26] methods for differential evolution; the social learning [27] and pairwise competition [28] methods for particle swarm optimization; and the multiple trajectory search [29] as well as multiple offspring sampling [30] methods for algorithm hybridization. Due to page limits, we cannot describe these techniques in detail.

\section{B. Decomposition Methods}

1) Interacting decision variables: In this sub-section, we start by presenting a formal definition of 'variable interactions', as some of the decomposition methods discussed below rely on variable interactions.

In an optimization problem, two decision variables interact if they cannot be optimized independently to find the global optimum. It is important to note that the interaction between given decision variables may be complicated. Take the following objective function as an example:

$$
f(\mathbf{x}):=x_{1}^{2}+\left(x_{2}-x_{3}\right)^{2}+\left(x_{3}-x_{4}\right)^{2}, \quad \mathbf{x} \in[-1,1]^{4},
$$

where ':=' denotes 'defined as'. Decision variable $x_{2}$ interacts with $x_{3}$, and $x_{3}$ interacts with $x_{4}$. Therefore, $x_{2}$ and $x_{4}$ are linked by $x_{3}$. If the optimal value of $x_{3}$ is known, $x_{2}$ and $x_{4}$ are independent, as they can be optimized separately. However, if $x_{3}$ needs to be optimized, $x_{2}$ and $x_{4}$ will influence each other. Therefore, $x_{2}$ conditionally interacts with $x_{4}{ }^{1}$. Note that conditional interaction only exists in overlapping problems e.g., Rosenbrock's function [21]. The formal definitions of interaction and conditional interaction are consistent with the definitions of direct interaction and indirect interaction described in [15], [31]:

\footnotetext{
${ }^{1}$ In [4], the relationships between $\left\{x_{2}, x_{4}\right\}$ and $\left\{x_{1}, x_{4}\right\}$ are both defined as additively separable. In this paper, we use conditional interaction to differentiate these two relationships.
} 
Definition 1. Let $f: \mathbb{R}^{n} \rightarrow \overline{\mathbb{R}}$ be a differentiable function. Decision variables $x_{i}$ and $x_{j}$ interact if a candidate solution $\mathrm{x}^{*}$ exists, such that

$$
\frac{\partial^{2} f\left(\mathbf{x}^{*}\right)}{\partial x_{i} \partial x_{j}} \neq 0 .
$$

denoted by $x_{i} \leftrightarrow x_{j}$. Decision variables $x_{i}$ and $x_{j}$ conditionally interact if for any candidate solution $\mathrm{x}^{*}$,

$$
\frac{\partial^{2} f\left(\mathbf{x}^{*}\right)}{\partial x_{i} \partial x_{j}}=0
$$

and a set of decision variables $\left\{x_{k 1}, \ldots, x_{k t}\right\} \subset X$ exists, such that $x_{i} \leftrightarrow x_{k 1} \leftrightarrow \ldots \leftrightarrow x_{k t} \leftrightarrow x_{j}$. Decision variables $x_{i}$ and $x_{j}$ are independent if for any candidate solution $\mathrm{x}^{*}, E q$. (3) holds and a set of decision variables $\left\{x_{k 1}, \ldots, x_{k t}\right\} \subset X$ does not exist, such that $x_{i} \leftrightarrow x_{k 1} \leftrightarrow \ldots \leftrightarrow x_{k t} \leftrightarrow x_{j}$.

2) Manual Decomposition: In these methods, the number of sub-components and the size of each sub-component are manually designed. These methods work well when combined with algorithms to solve fully separable problems. However, the performance deteriorates quickly when applied on partially separable problems or fully non-separable problems. The main reason is that it does not take the underlying structure of variable interactions into consideration.

Probably the first and simplest decomposition is the univariable grouping [9] method, which decomposes an $n$ dimensional problem into $n$ 1-dimensional sub-components. The uni-variable grouping method improved the performance of a genetic algorithm (GA) when solving benchmark separable problems, however it degraded the GA's performance when solving a benchmark non-separable problem - the Rosenbrock's function [9]. This performance difference may be attributed to the fact that the uni-variable grouping method decomposes an optimization problem without considering the interaction between decision variables.

The $S_{k}$ grouping [32] method is more flexible than the univariable grouping method when used to decompose an optimization problem. It decomposes an $n$-dimensional problem into $k s$-dimensional sub-components, $s<n$. The $S_{k}$ grouping method has been shown to be able to improve the performance of a particle swarm optimization [32] algorithm and a biogeograph based optimization [33] algorithm. However, like the uni-variable grouping method, the $S_{k}$ grouping method does not take variable interactions into consideration.

The random grouping ( $R G$ ) method is proposed within the context of a differential evolution cooperative co-evolution (DECC) framework [19]. It randomly assigns decision variables to predetermined number of sub-components before each evolutionary cycle. The RG method has been successfully applied to improve the performance of a particle swarm optimization [34] algorithm and an artificial bee colony [35] algorithm. However, it has been shown that the probability of assigning more than two interacting decision variables into one sub-component using the RG method is low [36]. Another limitation of $\mathrm{RG}$ is the requirement of setting an appropriate sub-component size. To address this issue, the multilevel cooperative co-evolution [37] algorithm takes the sub-component size as a parameter, and selects an appropriate sub-component size according to the historical performance.

A more sophisticated method - delta grouping [38] identifies variable interactions based on the averaged difference in a certain decision variable across the whole population. It generally outperforms the RG method when incorporated with the DECC framework to solve the CEC'2010 benchmark problems [38]. However on benchmark problems with more than one non-separable sub-component, the performance of the delta grouping method is low [10].

The $k$-means grouping [39] method uses a $k$-means clustering algorithm to construct decision variable groups. The decision variables with similar effects on the fitness value are placed into the same sub-component. The sub-component with the greatest contribution to the fitness value will be optimized with more iterations (FEs). The idea is similar to the contribution based cooperative co-evolution [23], [40] framework. Unlike most decomposition methods which group decision variables based on variable interactions, the $k$-means grouping method groups decision variables based on their contribution to the fitness improvement. Therefore, it is specifically tailored for problems with unbalanced sub-components [4], [39].

3) Automatic Decomposition: In these methods, the interacting decision variables are identified and automatically placed into the same sub-component. It is important to note that automatic decomposition considers the underlying variable interaction structure encapsulated within the search landscape. The decomposition method proposed in Section III falls into this category.

A representative automatic decomposition method - cooperative co-evolution with variable interaction learning (CCVIL) [41] - identifies the pairwise interaction between decision variables by the non-monotonicity detection. If the monotonicity of the fitness function with respect to $x_{i}$ does not change for different value of $x_{j}, x_{i}$ and $x_{j}$ are independent. Otherwise, decision variables $x_{i}$ and $x_{j}$ interact. The rationale behind the CCVIL method is consistent with the linkage identification by non-monotonicity detection [42] method. The CCVIL method is more accurate than most of the manual decomposition methods when identifying variable interactions. However, it still can not obtain acceptable results when used to decompose some benchmark problems [10].

The statistical variable interdependence learning (SL) [43] method identifies variable interactions based on the nonmonotonicity detection as well. Unlike the CCVIL method, the SL method detects the monotonicity relationship between $x_{i}$ and $x_{j}$ multi-times. The probability $\left(p_{i j}\right)$ of the observation of non-monotonicity is calculated. If the probability $p_{i j}$ is greater than a given threshold, decision variables $x_{i}$ and $x_{j}$ interact. The main issue of the SL method is the high computational complexity. The number of FEs needed to decompose an $n$ dimensional optimization problem is $4 m n^{2}$, where $m$ is the number of non-monotonicity detection conducted for each pair of decision variables.

To address the high computational complexity of the SL method, a fast variable interdependence searching [44] method is proposed. It identifies interactions between two subsets of decision variables, instead of two decision variables, by non- 
monotonicity detection. Therefore, it speeds up the decomposition process. The computational cost to decompose an $n$ dimensional problem can be reduced to $4 m n \log (n)$ in the worst case.

The differential grouping (DG) [10] method identifies variable interactions by detecting the fitness changes when perturbing the decision variables. If the fitness change induced by perturbing decision variable $x_{i}$ varies for different value of $x_{j}, x_{i}$ and $x_{j}$ interact. The rationale behind the DG method is consistent with the linkage identification by nonlinearity check [45] method. The DG method outperformed the CCVIL method when used to decompose the CEC'2010 benchmark problems [10]. However it has been shown not to be able to completely identify interacting decision variables in overlapping problems [15], [46].

Subsequently, the extended differential grouping (XDG) [15] method was proposed to address this issue, by placing all the linked (interacting and conditionally interacting) decision variables into one sub-component. It employs the same technique as DG to identify interacting decision variables. Then the overlapping between sub-components are checked to identify conditional interactions. However, the number of FEs used by XDG to decompose an $n$-dimensional problem is usually around $n^{2}$. The high complexity of the XDG method results in an inappropriate allocation of computational budget between decomposition and optimization, and prevents it from being applied to solve even higher dimensional problems.

The computational cost in the decomposition stage can be reduced to $\left(n^{2}+3 n+2\right) / 2$ by using the global differential grouping (GDG) [46] method. It employs the same technique as DG to identify the pairwise interactions between decision variables. The variable interaction matrix is calculated, which is regarded as the adjacency matrix of a graph. Then the depthfirst search or breadth-first search can be used to identify the connected components. Note that both the interacting and conditionally interacting decision variables will also be placed into one connected component (sub-component).

Recently, it has been shown that the minimal number of FEs used to identify the complete variable interaction matrix based on $\mathrm{DG}$ is $\left(n^{2}+n+2\right) / 2$ [47]. However, it may not need the entire variable interaction matrix to identify the connected components (sub-components). For example, if decision variable $x_{1}$ interacts with $x_{2}$ and $x_{3}$, the interaction between $x_{2}$ and $x_{3}$ needs not to be checked, as they belong to the same connected component.

The fast interdependency identification (FII) [48] method can further improve the efficiency of problem decomposition by avoiding the need to identify the complete variable interaction matrix. FII firstly identifies the separable decision variables by examining the interaction between one decision variable and the other variables. Then the interaction between non-separable decision variables is examined, and all the linked (connected) decision variables are placed into the same sub-component. The FII method is efficient when used to decompose benchmark problems with a large portion of separable decision variables. However on benchmark problems with conditional variable interactions, the number of FEs used by FII may still be in the magnitude of $n^{2}\left(\Theta\left(n^{2}\right)\right)$. In the next section, we will propose an efficient and robust method that can decompose any $n$-dimensional problem using less than $6 n \log _{2}(n)$ FEs.

\section{RECURSIVE DIFFERENTIAL GROUPING}

In this section, the proposed decomposition method - Recursive Differential Grouping (RDG) - is described in detail. Then, the computational complexity of the RDG method is presented.

Notation. Let $X$ be the set of decision variables $\left\{x_{1}, \ldots, x_{n}\right\}$; $U_{X}$ be the set of unit vectors in the decision space $R^{n}$. Let $X_{1}$ be a subset of decision variables $X_{1} \subset X$; and $U_{X_{1}}$ be a subset of $U_{X}$ such that any unit vector $\mathbf{u}=\left(u_{1}, \ldots, u_{n}\right) \in$ $U_{X_{1}}$, we have

$$
u_{i}=0, \quad \text { if } x_{i} \notin X_{1} .
$$

Directional Derivative. Let $f: \mathbb{R}^{n} \rightarrow \overline{\mathbb{R}}$ be a differentiable function, and $\mathbf{u}=\left(u_{1}, \ldots, u_{n}\right)$ be a vector from $U_{X}$. The directional derivative of $f$ in the direction $\mathbf{u}$, denoted $\mathcal{D}_{\mathbf{u}} f(\mathbf{x})$, is given by

$$
\mathcal{D}_{\mathbf{u}} f(\mathbf{x})=\sum_{i=1}^{n} \frac{\partial f(\mathbf{x})}{\partial x_{i}} u_{i}
$$

Proposition 1. Let $f: \mathbb{R}^{n} \rightarrow \overline{\mathbb{R}}$ be a differentiable function; $X_{1} \subset X$ and $X_{2} \subset X$ be two mutually exclusive subsets of decision variables: $X_{1} \cap X_{2}=\emptyset$. If there exist two unit vectors $\mathbf{u}_{1} \in U_{X_{1}}$ and $\mathbf{u}_{2} \in U_{X_{2}}$, and a candidate solution $\mathbf{x}^{*}$ in the decision space such that

$$
\mathcal{D}_{\mathbf{u}_{1}} \mathcal{D}_{\mathbf{u}_{2}} f\left(\mathbf{x}^{*}\right) \neq 0,
$$

there is some interaction between decision variables in $X_{1}$ and $X_{2}$.

Proof. Without loss of generality, we assume that $X_{1}=$ $\left\{x_{1,1}, \ldots, x_{1, p}\right\}, X_{2}=\left\{x_{2,1}, \ldots, x_{2, q}\right\}$, where $p, q$ are the number of decision variables in $X_{1}$ and $X_{2}$ respectively; $\mathbf{u}_{1}=\left(u_{1}^{1}, \ldots, u_{n}^{1}\right)$ and $\mathbf{u}_{2}=\left(u_{1}^{2}, \ldots, u_{n}^{2}\right)$. According to Directional Derivative,

$$
\mathcal{D}_{\mathbf{u}_{1}} \mathcal{D}_{\mathbf{u}_{2}} f(\mathbf{x})=\sum_{i=1}^{n} \sum_{j=1}^{n} \frac{\partial^{2} f(\mathbf{x})}{\partial x_{i} \partial x_{j}} u_{i}^{1} u_{j}^{2} .
$$

As $\mathbf{u}_{1}$ and $\mathbf{u}_{2}$ are two unit vectors from $U_{X_{1}}$ and $U_{X_{2}}$ respectively, we can obtain that:

$$
\begin{array}{cc}
u_{i}^{1}=0, & \text { if } x_{i} \notin X_{1}, \\
u_{j}^{2}=0, & \text { if } x_{j} \notin X_{2} .
\end{array}
$$

Therefore,

$$
\mathcal{D}_{\mathbf{u}_{1}} \mathcal{D}_{\mathbf{u}_{2}} f(\mathbf{x})=\sum_{i=1}^{p} \sum_{j=1}^{q} \frac{\partial^{2} f(\mathbf{x})}{\partial x_{1, i} \partial x_{2, j}} u_{1, i}^{1} u_{2, j}^{2},
$$

If (6) holds,

$$
\sum_{i=1}^{p} \sum_{j=1}^{q} \frac{\partial^{2} f\left(\mathbf{x}^{*}\right)}{\partial x_{1, i} \partial x_{2, j}} u_{1, i}^{1} u_{2, j}^{2} \neq 0 .
$$

Therefore, there exists at least one pair of $(i, j)$, such that

$$
\frac{\partial^{2} f\left(\mathbf{x}^{*}\right)}{\partial x_{1, i} \partial x_{2, j}} \neq 0 \text {. }
$$


Based on Definition 1, at least one pair of decision variables $x_{1, i} \in X_{1}$ and $x_{2, j} \in X_{2}$ interact.

Corollary 1. Let $f: \mathbb{R}^{n} \rightarrow \overline{\mathbb{R}}$ be an objective function; $X_{1} \subset$ $X$ and $X_{2} \subset X$ be two mutually exclusive subsets of decision variables: $X_{1} \cap X_{2}=\emptyset$. If there exist two unit vectors $\mathbf{u}_{1} \in$ $U_{X_{1}}$ and $\mathbf{u}_{2} \in U_{X_{2}}$, two real numbers $l_{1}, l_{2}>0$, and $a$ candidate solution $\mathrm{x}^{*}$ in the decision space, such that

$$
f\left(\mathbf{x}^{*}+l_{1} \mathbf{u}_{1}+l_{2} \mathbf{u}_{2}\right)-f\left(\mathbf{x}^{*}+l_{2} \mathbf{u}_{2}\right) \neq f\left(\mathbf{x}^{*}+l_{1} \mathbf{u}_{1}\right)-f\left(\mathbf{x}^{*}\right),
$$

there is some interaction between decision variables in $X_{1}$ and $X_{2}$.

Proof. With Proposition 1, we only need to prove the following statement:

Statement 1. If there exist two unit vectors $\mathbf{u}_{1} \in U_{X_{1}}$ and $\mathbf{u}_{2} \in U_{X_{2}}$, two real numbers $l_{1}, l_{2}>0$, and a candidate solution $\mathrm{x}^{*}$ in the decision space, such that Eq. (13) holds, then Eq. (6) is true.

It is equivalent to prove its contraposition:

Statement 2. If for any two unit vectors $\mathbf{u}_{1} \in U_{X_{1}}$ and $\mathbf{u}_{2} \in$ $U_{X_{2}}$, and for any candidate solution $\mathrm{x}^{*}$ in the decision space, the following condition holds:

$$
\mathcal{D}_{\mathbf{u}_{1}} \mathcal{D}_{\mathbf{u}_{2}} f\left(\mathbf{x}^{*}\right)=0,
$$

then

$$
f\left(\mathbf{x}^{*}+l_{1} \mathbf{u}_{1}+l_{2} \mathbf{u}_{2}\right)-f\left(\mathbf{x}^{*}+l_{2} \mathbf{u}_{2}\right)=f\left(\mathbf{x}^{*}+l_{1} \mathbf{u}_{1}\right)-f\left(\mathbf{x}^{*}\right),
$$

for any $l_{1}, l_{2}>0$.

In order to prove Statement 2, we first introduce line integral.

Line Integral. Let $L$ be a curve with end points $A$ and $B$ in the decision space $R^{n}$ and the arc length of $L$ be l. Let $C$ be any point on $L$ and the coordinate of $C(\mathrm{x})$ can be uniquely determined by the length of the arc $A C(s): \mathrm{x}=\mathrm{x}(s), s \in$ $[0, l]$. The integral of a function $g: \mathbb{R}^{n} \rightarrow \overline{\mathbb{R}}$ along the curve $L$ is given by

$$
\int_{L} g(\mathbf{x}) \mathrm{d} s=\int_{0}^{l} g(\mathbf{x}(s)) \mathrm{d} s .
$$

Let $A_{2}\left(\mathrm{x}^{*}\right)$ be any point in $R^{n}$, and $B_{2}$ be $\mathrm{x}^{*}+l_{2} \mathbf{u}_{2}$, where $\mathbf{u}_{2}$ is any vector in $U_{X_{2}}$ and $l_{2}$ is any positive real number. Let $C_{2}$ be any point on the segment $A_{2} B_{2}$. Therefore, the length of the segment $A_{2} B_{2}$ is $l_{2}$, and the coordinate of $C_{2}$ (x) can be uniquely determined by the length of the segment $A_{2} C_{2}$ $\left(s_{2}\right): \mathbf{x}\left(s_{2}\right)=\mathbf{x}^{*}+s_{2} \mathbf{u}_{2}, s_{2} \in\left[0, l_{2}\right]$. If Eq. (14) holds for any candidate solution in the decision space, then

$$
\mathcal{D}_{\mathbf{u}_{1}} \mathcal{D}_{\mathbf{u}_{2}} f(\mathbf{x})=0 \text {. }
$$

As $\mathcal{D}_{\mathbf{u}_{1}} \mathcal{D}_{\mathbf{u}_{2}} f(\mathbf{x})=\mathcal{D}_{\mathbf{u}_{2}} \mathcal{D}_{\mathbf{u}_{1}} f(\mathbf{x})$, by integrating both sides of Eq. (17) along the segment $A_{2} B_{2}$, we can obtain that

$$
\int_{0}^{l_{2}} \mathcal{D}_{\mathbf{u}_{1}} \mathcal{D}_{\mathbf{u}_{2}} f(\mathbf{x}) \mathrm{d} s_{2}=\int_{0}^{l_{2}} \mathcal{D}_{\mathbf{u}_{2}} \mathcal{D}_{\mathbf{u}_{1}} f(\mathbf{x}) \mathrm{d} s_{2}=0 .
$$

As

$$
\int_{0}^{l_{2}} \mathcal{D}_{\mathbf{u}_{2}}\left(\mathcal{D}_{\mathbf{u}_{1}} f\left(\mathbf{x}\left(s_{2}\right)\right)\right) \mathrm{d} s_{2}=\left.\mathcal{D}_{\mathbf{u}_{1}} f\left(\mathbf{x}\left(s_{2}\right)\right)\right|_{s_{2}=0} ^{s_{2}=l_{2}},
$$

thus

$$
\left.\mathcal{D}_{\mathbf{u}_{1}} f\left(\mathbf{x}\left(s_{2}\right)\right)\right|_{s_{2}=0} ^{s_{2}=l_{2}}=0
$$

and

$$
\mathcal{D}_{\mathbf{u}_{1}} f\left(\mathbf{x}^{*}+l_{2} \mathbf{u}_{2}\right)-\mathcal{D}_{\mathbf{u}_{1}} f\left(\mathbf{x}^{*}\right)=0 .
$$

As $A_{2}\left(\mathrm{x}^{*}\right)$ is any point in $R^{n}$, therefore

$$
\mathcal{D}_{\mathbf{u}_{1}} f\left(\mathbf{x}+l_{2} \mathbf{u}_{2}\right)=\mathcal{D}_{\mathbf{u}_{1}} f(\mathbf{x}) .
$$

Let $A_{1}\left(\mathrm{x}^{*}\right)$ be any point in $R^{n}$, and $B_{1}$ be $\mathbf{x}^{*}+l_{1} \mathbf{u}_{1}$, where $\mathbf{u}_{1}$ is any vector in $U_{X_{1}}$ and $l_{1}$ is any positive real number. Let $C_{1}$ be any point on the segment $A_{1} B_{1}$. Therefore, the length of the segment $A_{1} B_{1}$ is $l_{1}$, and the coordinate of $C_{1}$ (x) can be uniquely determined by the length of the segment $A_{1} C_{1}$ $\left(s_{1}\right): \mathbf{x}\left(s_{1}\right)=\mathbf{x}^{*}+s_{1} \mathbf{u}_{1}, s_{1} \in\left[0, l_{1}\right]$. Similarly, by integrating both sides of Eq. (22) along the segment $A_{1} B_{1}$, we can obtain

$$
\int_{0}^{l_{1}} \mathcal{D}_{\mathbf{u}_{1}} f\left(\mathbf{x}\left(s_{1}\right)+l_{2} \mathbf{u}_{2}\right) \mathrm{d} s_{1}=\int_{0}^{l_{1}} \mathcal{D}_{\mathbf{u}_{1}} f\left(\mathbf{x}\left(s_{1}\right)\right) \mathrm{d} s_{1} .
$$

Therefore,

$$
f\left(\mathbf{x}^{*}+l_{1} \mathbf{u}_{1}+l_{2} \mathbf{u}_{2}\right)-f\left(\mathbf{x}^{*}+l_{2} \mathbf{u}_{2}\right)=f\left(\mathbf{x}^{*}+l_{1} \mathbf{u}_{1}\right)-f\left(\mathbf{x}^{*}\right) .
$$

Thus, Statement 2 is proved, and Statement 1 and Corollary 1 are true.

With Corollary 1, the interaction between two subsets of decision variables $\left(X_{1}\right.$ and $\left.X_{2}\right)$ can be identified using the following procedures:

1) Set all the decision variables to the lower bounds (lb) of the search space $\left(\mathrm{x}_{l, l}\right)$;

2) Perturb the decision variables $X_{1}$ of $\mathbf{x}_{l, l}$ from the lower bounds to the upper bounds $(\mathbf{u b})$, denoted by $\mathbf{x}_{u, l}$;

3) Calculate the fitness difference $\left(\delta_{1}\right)$ between $\mathbf{x}_{l, l}$ and $\mathbf{x}_{u, l}$;

4) Perturb the decision variables $X_{2}$ of $\mathbf{x}_{l, l}$ and $\mathbf{x}_{u, l}$ from the lower bounds to the middle between the lower bounds and upper bounds, denoted by $\mathbf{x}_{l, m}$ and $\mathbf{x}_{u, m}$ respectively;

5) Calculate the fitness difference $\left(\delta_{2}\right)$ between $\mathbf{x}_{l, m}$ and $\mathbf{x}_{u, m}$

6) If the difference between $\delta_{1}$ and $\delta_{2}$ is greater than a threshold $\epsilon$, there is some interaction between $X_{1}$ and $X_{2}$.

The two subscripts of $\mathrm{x}$ denote the values of $X_{1}$ and $X_{2}$ respectively: ' $l$ ' means lower bounds, ' $u$ ' means upper bounds, and ' $m$ ' means the middle between the lower bounds and upper bounds. The threshold $\epsilon$ is estimated based on the magnitude of the objective space [46]:

$$
\epsilon=\alpha \cdot \min \left\{\left|f\left(\mathbf{x}_{1}\right)\right|, \cdots,\left|f\left(\mathbf{x}_{k}\right)\right|\right\},
$$

where $\mathbf{x}_{1}, \cdots, \mathbf{x}_{k}$ are $k$ randomly generated candidate solutions, and $\alpha$ is the control coefficient [46].

Based on Corollary 1, we propose the Recursive Differential Grouping (RDG) (Algorithm 1) method to efficiently decompose an optimization problem. The decomposition by the RDG method considers the underlying structure of variable interactions. Taking the following objective function as an example:

$$
f(\mathbf{x}):=x_{1}^{2}+\left(x_{2}-x_{3}\right)^{2}+\left(x_{3}-x_{4}\right)^{2}+\left(x_{5}-x_{6}\right)^{2}, \mathbf{x} \in[-1,1]^{6},
$$




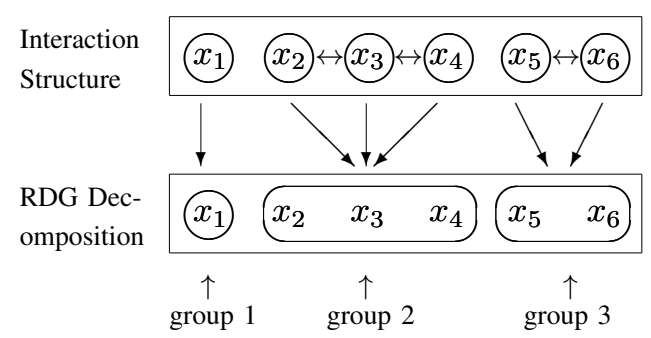

Fig. 2. The variable interaction structure and the RDG decomposition of the objective function given in Eq. (26). The notation $x_{i} \leftrightarrow x_{j}$ denotes that decision variable $x_{i}$ interacts with $x_{j}$.

the decision variables $\left(x_{2}, x_{3}, x_{4}\right)$ interact, as well as $\left(x_{5}, x_{6}\right)$. Therefore, the decomposition by the RDG method is $\left\{\left(x_{1}\right),\left(x_{2}, x_{3}, x_{4}\right),\left(x_{5}, x_{6}\right)\right\}$, as shown in Fig. 2.

The inputs to the RDG method are the fitness function $(f)$, the upper bounds (ub), the lower bounds (lb), and the threshold $(\epsilon)$ which is estimated using Eq. (25). The outputs are the separable variable group (seps) and the non-separable variable groups (nonseps). The seps contains one group of all separable decision variables. The nonseps contains several groups of non-separable decision variables. Each group of decision variables will form a sub-component.

The RDG method begins by identifying the interaction between the first decision variable $x_{1}$ and the remaining decision variables. If no interaction is detected, $x_{1}$ will be placed in the separable decision variable group, and the algorithm will move on to the next decision variable $x_{2}$. If any interaction is detected, the remaining decision variables will be divided into two (nearly) equally-sized groups $G_{1}$ and $G_{2}$. Then the interaction between $x_{1}$ and $G_{1}, x_{1}$ and $G_{2}$ will be identified respectively. This process is recursively conducted until all the individual decision variables that interact with $x_{1}$ are identified and placed in the decision variable subset $X_{1}$ with $x_{1}$.

Then, the RDG method examines the interaction between $X_{1}$ and the remaining decision variables (excluding the decision variables in $X_{1}$ ) to identify the individual decision variables that conditionally interact with $x_{1}$ (linked by other decision variables). If any interaction is identified, the interacting decision variables will be placed into $X_{1}$. This process is repeated until no interaction can be further detected between $X_{1}$ and the remaining decision variables (exclusive $X_{1}$ ). The decision variables in $X_{1}$ will be placed in a non-separable group.

The RDG method moves on to the next decision variable that has not been grouped $\left(x_{i}\right)$. The interaction between $x_{i}$ and the remaining decision variables will be examined, and both the interacting and conditionally interacting (linked) decision variables will be placed into one group with $x_{i}$. This process is repeated until all of the decision variables are grouped. It returns the separable (seps) and the non-separable (nonseps) decision variable groups as the outputs.

The computational complexity of the RDG method when used to decompose an $n$-dimensional problem is $\mathcal{O}(n \log (n))$, which is analyzed as follows:

1) When decomposing an $n$-dimensional fully separable problem, the computational complexity of the RDG
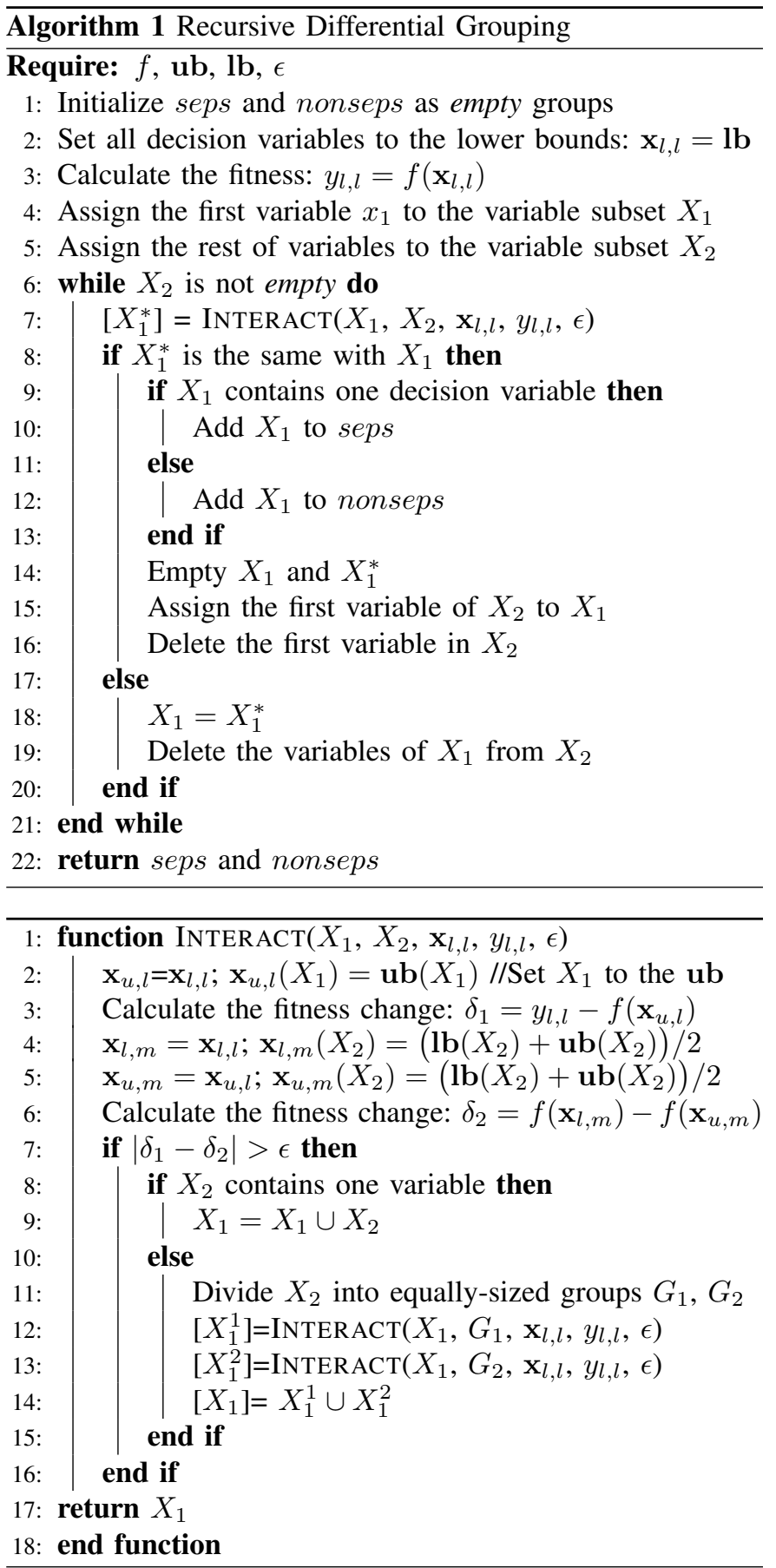

method is $\Theta(n)$ in terms of the number of FEs. For each decision variable, 3 FEs are used to determine its separability. Therefore, totally about $3 n$ FEs are needed.

2) When decomposing an $n$-dimensional fully non-separable problem with one sub-component, the computational complexity of the RDG method is $\Theta(n)$. When grouping the $n$ interacting decision variables, the function 'INTERACT' is executed about $\sum_{i=0}^{k}\left(n / 2^{i}\right)$ times, where $k=\log _{2}(n)$.

$$
\sum_{i=0}^{k} \frac{n}{2^{i}}=n\left(2-\left(\frac{1}{2}\right)^{k}\right)<2 n
$$

It consumes 3 FEs each time the function 'INTERACT' is 
executed. Therefore totally about $6 n$ FEs are used.

3) When decomposing an $n$-dimensional partially separable problem with $n / m$ sub-components, the computational complexity of the RDG method is $\Theta(n \log (n))$, where $n$ is the dimensionality and $m$ is the number of decision variables in each sub-component. When grouping $m$ interacting decision variables into one sub-component, the function 'INTERACT' is executed less than $2 m \times$ $\log _{2}(n)$ times, each time consuming 3 FEs. The number of sub-components is $n / m$. Therefore, totally less than $3 \times 2 m \times \log _{2}(n) \times n / m=6 n \log _{2}(n)$ FEs are used.

4) When decomposing an $n$-dimensional partially separable problem with an $m$-dimensional non-separable subcomponent, the computational complexity of the RDG method is $\mathcal{O}(\max \{n, m \log (n)\})$. The RDG algorithm consumes about $3(n-m)$ FEs to identify the $n-m$ separable decision variables, and consumes less than $6 m \times \log _{2}(n)$ FEs to identify the $m$ interacting decision variables. Therefore, totally less than $3(n-m)+6 m \times$ $\log _{2}(n)$ FEs are used.

5) When decomposing an $n$-dimensional overlapping problem (e.g., Rosenbrock's function [21]), the computational complexity of the RDG method is $\Theta(n \log (n))$. Starting from $x_{1}$, it consumes about $3 \times 2 \times 2 \log _{2}(n)=12 \log _{2}(n)$ FEs to identify the two decision variables $\left(x_{p}\right.$ and $\left.x_{q}\right)$ that interact with $x_{1}$. Then it also consumes about $12 \log _{2}(n)$ FEs to identify the two decision variables that interact with $\left(x_{p}, x_{1}, x_{q}\right)$. Therefore, totally about $n / 2 \times 12 \log _{2}(n)=6 n \log _{2}(n)$ FEs are used.

\section{ExPERIMENTAL Methodology}

In this section, comprehensive numerical experiments are designed to evaluate the proposed RDG method. Two research questions guide the experimental design to evaluate the efficacy of the proposed RDG method:

Q1. Can the proposed RDG method decompose the CEC'2010 and CEC'2013 benchmark problems more efficiently when compared against other well-known decomposition methods?

Q2. Can the proposed RDG method outperform other wellknown decomposition methods when embedded into a CC framework to solve the CEC'2010 and CEC'2013 benchmark problems?

To answer Q1, the proposed RDG method was used to decompose the CEC'2010 [20] and CEC'2013 [21] benchmark problems ${ }^{2}$. Two metrics were employed to evaluate the performance of a decomposition method: (1) the number of FEs used to decompose the problem; and (2) the percentage of interacting decision variables that are correctly grouped, defined as

Decomposition Accuracy. Let $G=\left\{g_{1}, \ldots, g_{m}\right\}$ denote the groups of interacting decision variables in a problem $f$, and $\tilde{G}=\left\{\tilde{g}_{1}, \ldots, \tilde{g}_{n}\right\}$ denote the groups of interacting decision variables that are identified by a decomposition method. Let

\footnotetext{
${ }^{2}$ The MATLAB implementation of the RDG method can be accessed from the following link: https://bitbucket.org/yuans/rdg.
}

TABLE I

THE PARAMETER SETTINGS FOR ALL THE DECOMPOSITION METHODS USED IN THE EXPERIMENTS. DG2 IS A PARAMETER-FREE METHOD.

\begin{tabular}{ll}
\hline Decomposition Methods & Parameter Settings \\
\hline RDG & control coefficient $\alpha=10^{-12}$ and $k=10$ \\
GDG & control coefficient $\alpha=10^{-12}$ and $k=10$ \\
XDG & threshold $\epsilon=10^{-1}$ \\
DG & threshold $\epsilon=10^{-3}$ \\
DG2 & parameter free \\
FII & threshold $\epsilon=10^{-2}$ \\
D (delta grouping) & sub-component size sub_dim $=100$ \\
RG & sub-component size sub_dim $=100$ \\
\hline
\end{tabular}

$G_{i}=\left\{g_{i, 1}, \ldots, g_{i, m}\right\}$ denote the $i_{\text {th }}$ permutation of $G$, where $1 \leq i \leq m !$, and $\tilde{G}_{j}=\left\{\tilde{g}_{j, 1}, \ldots, \tilde{g}_{j, n}\right\}$ denote the $j_{t h}$ permutation of $\tilde{G}$, where $1 \leq j \leq n$ !. The decomposition accuracy (DA) of the decomposition method on $f$ is defined as

$$
D A=\frac{\max _{i, j}\left\{\sum_{k=1}^{\min \{m, n\}}\left|g_{i, k} \cap \tilde{g}_{j, k}\right|\right\}}{\sum_{i=1}^{m}\left|g_{i}\right|}
$$

where $\left|g_{i}\right|$ denotes the number of decision variables in $g_{i}$.

The performance of the RDG method was then compared to the GDG [46], XDG [15], DG [10], as well as two recently published methods - DG2 [47], and FII [48]. The parameter settings for all the decomposition methods used in the experiments are shown in Table I. The threshold values $(\epsilon)$ estimated by the RDG (or GDG) method for each problem were recorded. Note that the RDG and GDG methods use the same approach (Eq. (25)) to estimate the threshold values.

To answer Q2, the proposed RDG method was embedded into the DECC [19] / CMAESCC [46] framework to solve the CEC'2010 and CEC'2013 benchmark problems. The DECC is the most widely used $\mathrm{CC}$ framework, which employs a variant of Differential Evolution - SaNSDE [49] - to solve each subcomponent cooperatively. The CMAESCC framework uses the well-known CMA-ES [50] algorithm to solve each subcomponent. It performs well when used to solve the CEC'2010 and CEC'2013 benchmark problems. The parameter settings for the DECC and CMAESCC frameworks were consistent with the original papers. The maximum number of FEs was set to $3 \times 10^{6}$, divided between the decomposition stage and optimization stage. For each benchmark problem, the median, mean and standard deviation of the best solutions found by the DECC/CMAESCC-RDG algorithm based on 25 independent runs were recorded. The performance of the RDG method was compared against the performance of the XDG, GDG, DG, DG2, FII, as well as two manual decomposition methods - D (delta grouping [38]) and RG [19] methods, when embedded in each CC framework.

The performance of DECC/CMAESCC-RDG was also compared to the performance of two state-of-the-art hybrid algorithms - MOS [30] and MA-SW-Chains [51] with default parameter settings. The MOS algorithm evaluates the constituent algorithms in each generation, and the better performed constituent algorithm will be used to generate more offspring. 
MOS achieved the best performance in the 2011 special issue of the Soft Computing journal. The MA-SW-Chains algorithm assigns to each individual a local search intensity that depends on its features, by chaining different local search applications. MA-SW-Chains achieved the best performance in the CEC 2010 special session and competition on LSGO.

The Kruskal-Wallis nonparametric one-way ANOVA test [52] with $95 \%$ confidence interval was used to determine whether the performance of at least one algorithm was significantly different from the others. Then a series of Wilcoxon rank-sum tests (significance level $\alpha=0.05$ ) with Holm p-value correction [52] was conducted in a pairwise fashion to find the better performing algorithm.

\section{EXPERIMENTAL RESULTS}

Comprehensive experimental results are presented and discussed in this section. Section V-A presents the decomposition comparison between the RDG method and five other methods, thus addressing Q1. Section V-B presents the optimization comparison between the RDG method and seven other methods when embedded into the DECC/CMAESCC framework to solve the benchmark problems, thus addressing Q2.

\section{A. Decomposition Comparison}

Table II lists the decomposition results of the RDG, GDG, XDG and DG methods on the CEC'2010 and CEC'2013 benchmark problems. The parameter settings for the four decomposition methods are consistent with Table I. In Table II, "DA" represents the decomposition accuracy - the percentage of interacting decision variables that correctly grouped; "FEs" represents the number of FEs used in the decomposition stage; " $\epsilon$ " represents the threshold used to identify interactions between decision variables. Note that the threshold values used by RDG are the same as those used by GDG. The entries with the best decomposition accuracy achieved using the smallest number of FEs are highlighted in bold. Different categories of benchmark problems are divided by lines.

The RDG and GDG methods obtain nearly the same decomposition accuracy across all the benchmark problems. The reason for this is that RDG uses the same approach (Eq. (25)) as GDG to estimate the threshold values. However, RDG is much more efficient than GDG in terms of FEs used. Note that the number of FEs used by GDG to decompose an $n$ dimensional problem is fixed: $\left(n^{2}+3 n+2\right) / 2$.

The first three problems $\left(f_{1}-f_{3}\right)$ from each benchmark suite are fully separable. Therefore, decomposition accuracy is not applicable to these problems. On $f_{1}$ and $f_{2}$ (CEC'2010 and CEC'2013), the RDG method successfully identifies all the decision variables as separable, using a small number of FEs. However on $f_{3}$, the RDG and GDG methods identify all the separable variables as non-separable, and place them into one sub-component. The reason for this is that the threshold value is under-estimated by the RDG and GDG methods on $f_{3}$.

The CEC'2013 $f_{13}$ and $f_{14}$ are benchmark problems with overlapping (conforming or conflicting) sub-components. It is not clear yet what is the best approach to decompose these problems [4], [21]. The RDG, GDG and XDG methods place all the overlapped sub-components into one group. On the other benchmark problems where the sub-components are independent with each other, the 'ideal' decomposition can possibly be achieved (See [4], [10], [20], [21] for more information). Note that the $100 \%$ decomposition accuracy in Table II corresponds to the ideal decomposition.

On the CEC'2010 partially separable problems $\left(f_{4^{-}} f_{18}\right)$, the RDG method consistently achieves the best results when compared against GDG, XDG and DG. The RDG, GDG and XDG methods achieve the ideal decomposition on all of these benchmark problems. However, the number of FEs used by the GDG and XDG methods is usually several magnitude larger than that used by the RDG method. The DG method performs well on the benchmark problems without conditional variable interactions. On $f_{19}$, the DG method uses the smallest number of FEs to decompose the problem. However on problems with conditional variable interactions (overlapping problems e.g., CEC'2010 $f_{13}, f_{18}$ and $f_{20}$ ), the decomposition accuracy of the DG method is low. The reason for this is that DG is unable to completely identify variable interactions in overlapping problems [15], [46].

On the CEC'2013 partially separable problems $\left(f_{4}-f_{11}\right)$, the RDG method achieves the best results on 4 out of 8 benchmark problems. We observe that it is generally more difficult to identify the variable interactions in the CEC'2013 than the CEC'2010 benchmark problems. None of the four methods can perfectly decompose the CEC'2013 $f_{8}, f_{10}$ and $f_{11}$ problems. On CEC'2013 $f_{11}$, the threshold value is underestimated by the RDG and GDG methods (Eq. (25)), resulting in placing all the decision variables into a single non-separable group. While on CEC'2013 $f_{8}$ and $f_{10}$, the threshold value is overestimated, resulting in some omissions of variable interactions being identified.

The threshold values estimated by the GDG and RDG methods vary significantly across the CEC'2010 and CEC'2013 benchmark problems. Therefore, it is very difficult to find a single threshold value to accurately identify variable interactions across all the problems. Although the threshold value $\epsilon=10^{-1}$ works well for the XDG method on the CEC'2010 benchmark problems, it fails on some of the CEC'2013 benchmark problems e.g., $f_{5}-f_{8}$. On $f_{5}$, the XDG algorithm (with $\epsilon=10^{-1}$ ) identifies all the interacting decision variables as separable. The reason for this is that the threshold value $10^{-1}$ is too large compared with the computational error, which is equal to $8.03 \times 10^{-5}$ estimated by the RDG and GDG methods. When using the estimated computational error as the threshold value, the XDG method achieves equal decomposition accuracy with the RDG method.

To further show the efficacy of the RDG method, we compare the performance of RDG against two recently published methods - DG2 and FII. The average number of FEs used by RDG to decompose the CEC'2010 and CEC'2013 benchmark problems is $1.47 \times 10^{4}$, which is less than that used by the DG2 and FII methods: $4.95 \times 10^{5}$ and $4.94 \times 10^{4}$ respectively. The detailed decomposition results of the DG2 and FII methods are presented in the supplementary documents.

The DG2 method uses a fixed number of FEs to decompose an $n$-dimensional problem: $\left(n^{2}+n+2\right) / 2$, which has been 
TABLE II

THE EXPERIMENTAL RESULTS OF THE PROPOSED RDG METHOD WHEN USED TO DECOMPOSE THE CEC'2010 AND CEC'2013 BENCHMARK PROBLEMS "DA" IS THE DECOMPOSITION ACCURACY; "FES" IS THE FES USED; “ $\epsilon$ " IS THE THRESHOLD. NOTE THAT THE THRESHOLD VALUES USED BY RDG ARE THE SAME AS THOSE USED BY GDG. THE PERFORMANCE OF THE RDG METHOD IS COMPARED WITH THE PERFORMANCES OF THE GDG, XDG AND DG METHODS. THE ENTRIES WITH THE BEST DA ACHIEVED USING THE LOWEST FES ARE HIGHLIGHTED IN BOLD.

\begin{tabular}{|c|c|c|c|c|c|c|c|c|c|c|}
\hline \multirow{2}{*}{$\begin{array}{l}\text { Bench- } \\
\text { marks }\end{array}$} & \multirow{2}{*}{$\begin{array}{l}\text { Func } \\
\text { Num }\end{array}$} & \multicolumn{3}{|c|}{ RDG } & \multicolumn{2}{|l|}{ GDG } & \multicolumn{2}{|c|}{$\mathrm{XDG}\left(\epsilon=10^{-1}\right)$} & \multicolumn{2}{|c|}{ DG $\left(\epsilon=10^{-3}\right)$} \\
\hline & & DA & FEs & $\epsilon$ & DA & FEs & DA & FEs & DA & FEs \\
\hline \multirow{20}{*}{ CEC'2010 } & $f_{1}$ & - & $3.00 \mathrm{e}+03$ & $4.11 \mathrm{e}-01$ & - & $5.01 \mathrm{e}+05$ & - & $1.00 \mathrm{e}+06$ & - & $1.00 \mathrm{e}+06$ \\
\hline & $f_{2}$ & - & $3.00 \mathrm{e}+03$ & $2.49 \mathrm{e}-08$ & - & $5.01 \mathrm{e}+05$ & - & $1.00 \mathrm{e}+06$ & - & $1.00 \mathrm{e}+06$ \\
\hline & $f_{3}$ & - & $6.00 \mathrm{e}+03$ & $2.15 \mathrm{e}-11$ & - & $5.01 \mathrm{e}+05$ & - & $1.00 \mathrm{e}+06$ & - & $1.00 \mathrm{e}+06$ \\
\hline & $f_{4}$ & $100 \%$ & $4.20 \mathrm{e}+03$ & $1.03 \mathrm{e}+04$ & $100 \%$ & $5.01 e+05$ & $100 \%$ & $8.05 e+04$ & $100 \%$ & $1.45 e+04$ \\
\hline & $f_{5}$ & $100 \%$ & $4.15 e+03$ & $1.14 \mathrm{e}-03$ & $100 \%$ & $5.01 \mathrm{e}+05$ & $100 \%$ & $9.98 \mathrm{e}+05$ & $100 \%$ & $9.05 e+05$ \\
\hline & $f_{6}$ & $100 \%$ & $5.00 e+04$ & $2.13 \mathrm{e}-05$ & $100 \%$ & $5.01 \mathrm{e}+05$ & $100 \%$ & $9.98 \mathrm{e}+05$ & $100 \%$ & $9.06 e+05$ \\
\hline & $f_{7}$ & $100 \%$ & $4.23 e+03$ & $5.17 \mathrm{e}+00$ & $100 \%$ & $5.01 \mathrm{e}+05$ & $100 \%$ & $9.98 \mathrm{e}+05$ & $68.0 \%$ & $6.77 e+04$ \\
\hline & $f_{8}$ & $100 \%$ & $5.60 \mathrm{e}+03$ & $2.62 \mathrm{e}+05$ & $100 \%$ & $5.01 \mathrm{e}+05$ & $100 \%$ & $1.21 \mathrm{e}+05$ & $90.0 \%$ & $2.32 \mathrm{e}+04$ \\
\hline & $f_{9}$ & $100 \%$ & $1.40 \mathrm{e}+04$ & $4.88 \mathrm{e}-01$ & $100 \%$ & $5.01 \mathrm{e}+05$ & $100 \%$ & $9.77 \mathrm{e}+05$ & $100 \%$ & $2.70 \mathrm{e}+05$ \\
\hline & $f_{10}$ & $100 \%$ & $1.40 e+04$ & $2.52 \mathrm{e}-08$ & $100 \%$ & $5.01 \mathrm{e}+05$ & $100 \%$ & $9.77 e+05$ & $100 \%$ & $2.72 \mathrm{e}+05$ \\
\hline & $f_{11}$ & $100 \%$ & $1.36 \mathrm{e}+04$ & $2.36 \mathrm{e}-10$ & $100 \%$ & $5.01 \mathrm{e}+05$ & $100 \%$ & $9.78 \mathrm{e}+05$ & $99.8 \%$ & $2.70 \mathrm{e}+05$ \\
\hline & $f_{12}$ & $100 \%$ & $1.43 e+04$ & $4.26 \mathrm{e}-05$ & $100 \%$ & $5.01 \mathrm{e}+05$ & $100 \%$ & $9.77 \mathrm{e}+05$ & $100 \%$ & $2.71 e+05$ \\
\hline & $f_{13}$ & $100 \%$ & $2.92 \mathrm{e}+04$ & $3.71 \mathrm{e}+00$ & $100 \%$ & $5.01 \mathrm{e}+05$ & $100 \%$ & $1.00 \mathrm{e}+06$ & $31.8 \%$ & $5.03 e+04$ \\
\hline & $f_{14}$ & $100 \%$ & $2.05 e+04$ & $4.15 \mathrm{e}-01$ & $100 \%$ & $5.01 \mathrm{e}+05$ & $100 \%$ & $9.53 e+05$ & $100 \%$ & $2.10 \mathrm{e}+04$ \\
\hline & $f_{15}$ & $100 \%$ & $2.05 e+04$ & $2.53 \mathrm{e}-08$ & $100 \%$ & $5.01 \mathrm{e}+05$ & $100 \%$ & $9.53 e+05$ & $100 \%$ & $2.10 \mathrm{e}+04$ \\
\hline & $f_{16}$ & $100 \%$ & $2.09 \mathrm{e}+04$ & $4.30 \mathrm{e}-10$ & $100 \%$ & $5.01 \mathrm{e}+05$ & $100 \%$ & $9.56 \mathrm{e}+05$ & $99.6 \%$ & $2.11 e+04$ \\
\hline & $f_{17}$ & $100 \%$ & $2.07 e+04$ & $1.10 \mathrm{e}-04$ & $100 \%$ & $5.01 \mathrm{e}+05$ & $100 \%$ & $9.53 e+05$ & $100 \%$ & $2.10 \mathrm{e}+04$ \\
\hline & $f_{18}$ & $100 \%$ & $4.98 \mathrm{e}+04$ & $8.19 \mathrm{e}+00$ & $100 \%$ & $5.01 \mathrm{e}+05$ & $100 \%$ & $9.99 \mathrm{e}+05$ & $23.0 \%$ & $3.96 \mathrm{e}+04$ \\
\hline & $f_{19}$ & $100 \%$ & $6.00 \mathrm{e}+03$ & $6.14 \mathrm{e}-04$ & $100 \%$ & $5.01 \mathrm{e}+05$ & $100 \%$ & $3.99 \mathrm{e}+03$ & $100 \%$ & $2.00 \mathrm{e}+03$ \\
\hline & $f_{20}$ & $100 \%$ & $5.08 e+04$ & $8.53 e+00$ & $100 \%$ & $5.01 \mathrm{e}+05$ & $100 \%$ & $1.00 \mathrm{e}+06$ & $28.7 \%$ & $1.55 \mathrm{e}+05$ \\
\hline \multirow{15}{*}{ CEC'2013 } & $f_{1}$ & - & $3.00 \mathrm{e}+03$ & $4.20 \mathrm{e}-01$ & - & $5.01 \mathrm{e}+05$ & - & $1.00 \mathrm{e}+06$ & - & $1.00 \mathrm{e}+06$ \\
\hline & $f_{2}$ & - & $3.00 \mathrm{e}+03$ & $1.31 \mathrm{e}-07$ & - & $5.01 \mathrm{e}+05$ & - & $1.00 \mathrm{e}+06$ & - & $1.00 \mathrm{e}+06$ \\
\hline & $f_{3}$ & - & $6.00 \mathrm{e}+03$ & $2.16 \mathrm{e}-11$ & - & $5.01 \mathrm{e}+05$ & - & $1.00 \mathrm{e}+06$ & - & $1.00 \mathrm{e}+06$ \\
\hline & $f_{4}$ & $100 \%$ & $9.84 e+03$ & $7.22 \mathrm{e}+01$ & $100 \%$ & $5.01 \mathrm{e}+05$ & $33.3 \%$ & $3.97 \mathrm{e}+05$ & $95.3 \%$ & $1.56 \mathrm{e}+04$ \\
\hline & $f_{5}$ & $100 \%$ & $1.01 e+04$ & $8.03 e-05$ & $100 \%$ & $5.01 \mathrm{e}+05$ & $0.00 \%$ & $1.00 \mathrm{e}+06$ & $0.00 \%$ & $1.00 \mathrm{e}+06$ \\
\hline & $f_{6}$ & $100 \%$ & $1.32 \mathrm{e}+04$ & $1.07 \mathrm{e}-06$ & $100 \%$ & $5.01 \mathrm{e}+05$ & $50.0 \%$ & $9.90 \mathrm{e}+05$ & $82.6 \%$ & $5.79 \mathrm{e}+05$ \\
\hline & $f_{7}$ & $100 \%$ & $9.82 \mathrm{e}+03$ & $5.82 \mathrm{e}+05$ & $100 \%$ & $5.01 \mathrm{e}+05$ & $33.3 \%$ & $2.66 \mathrm{e}+04$ & $39.6 \%$ & $1.14 \mathrm{e}+04$ \\
\hline & $f_{8}$ & $80.0 \%$ & $1.95 \mathrm{e}+04$ & $1.20 \mathrm{e}+06$ & $80.0 \%$ & $5.01 \mathrm{e}+05$ & $10.0 \%$ & $6.83 e+04$ & $85.6 \%$ & $2.26 \mathrm{e}+04$ \\
\hline & $f_{9}$ & $100 \%$ & $1.92 \mathrm{e}+04$ & $6.07 \mathrm{e}-03$ & $100 \%$ & $5.01 \mathrm{e}+05$ & $99.9 \%$ & $9.35 e+05$ & $100 \%$ & $1.76 e+04$ \\
\hline & $f_{10}$ & $82.7 \%$ & $1.91 \mathrm{e}+04$ & $9.80 \mathrm{e}-05$ & $90.0 \%$ & $5.01 \mathrm{e}+05$ & $79.6 \%$ & $9.52 \mathrm{e}+05$ & $79.8 \%$ & $4.86 e+04$ \\
\hline & $f_{11}$ & $10.0 \%$ & $1.06 \mathrm{e}+04$ & $1.52 \mathrm{e}+06$ & $10.0 \%$ & $5.01 \mathrm{e}+05$ & $10.0 \%$ & $2.20 \mathrm{e}+04$ & $37.7 \%$ & $9.10 \mathrm{e}+03$ \\
\hline & $f_{12}$ & $100 \%$ & $5.08 e+04$ & $8.57 \mathrm{e}+00$ & $100 \%$ & $5.01 \mathrm{e}+05$ & $100 \%$ & $1.00 \mathrm{e}+06$ & $39.0 \%$ & $1.49 \mathrm{e}+05$ \\
\hline & $f_{13}$ & - & $8.39 \mathrm{e}+03$ & $1.83 e+06$ & - & $4.10 \mathrm{e}+05$ & - & $1.29 \mathrm{e}+04$ & - & $5.86 \mathrm{e}+03$ \\
\hline & $f_{14}$ & - & $1.61 \mathrm{e}+04$ & $5.45 e+06$ & - & $4.10 \mathrm{e}+05$ & - & $3.57 \mathrm{e}+04$ & - & $1.39 \mathrm{e}+04$ \\
\hline & $f_{15}$ & $100 \%$ & $6.16 \mathrm{e}+03$ & $2.70 \mathrm{e}+06$ & $100 \%$ & $5.01 \mathrm{e}+05$ & $100 \%$ & $3.99 \mathrm{e}+03$ & $100 \%$ & $2.00 \mathrm{e}+03$ \\
\hline
\end{tabular}

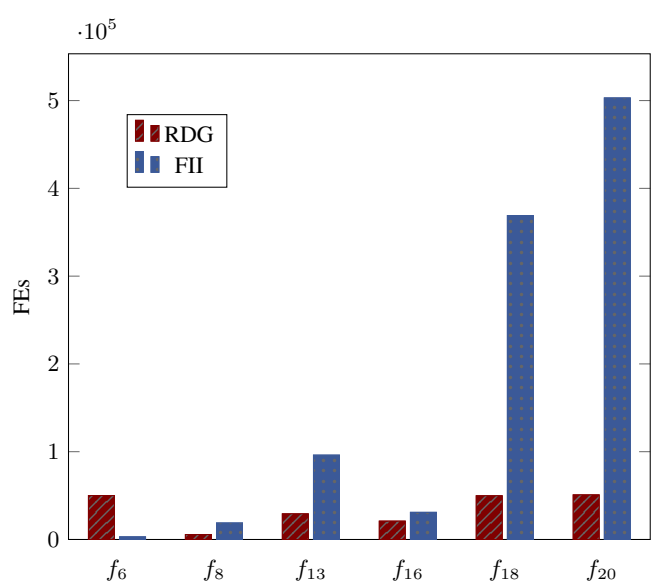

Fig. 3. The benchmark problems from the CEC'2010 test suite on which the RDG and FII methods generate significant different results (the difference between the number of FEs used is greater than $10^{4}$ ). shown to be the lower bound of identifying the complete variable interaction matrix. With the complete variable interaction matrix being identified, it is possible to generate an effective decomposition for the problems with overlapping sub-components, e.g., CEC'2013 $f_{13}$ and $f_{14}$ [47]. However, the existing automatic decomposition methods place all the linked decision variables into one sub-component.

DG2 is a parametric-free method, which automatically estimates the threshold values in the decomposition process. The decomposition accuracy of DG2 on the CEC'2010 and CEC'2013 benchmark problems is high. It achieves 100\% decomposition accuracy for the CEC'2013 $f_{10}$ and $f_{11}$ problems. The variable interactions in these two problems are difficult for the other decomposition methods to identify. However on CEC'2013 $f_{7}$ and $f_{8}$, the decomposition accuracy of DG2 is less than that of RDG.

The FII method performs well when used to decompose the benchmark problems with a large portion of separable decision 


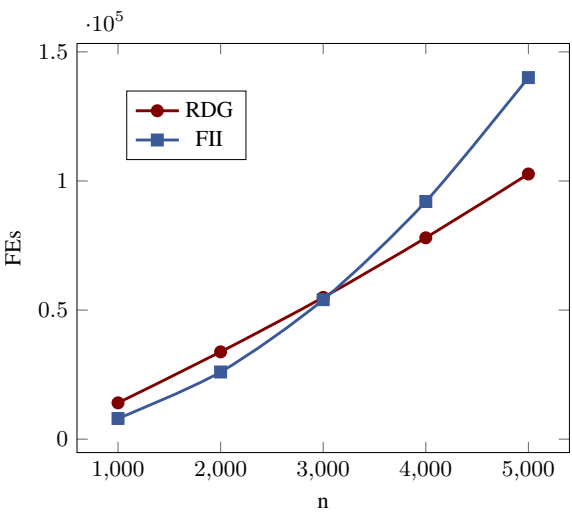

(a) CEC'2010 $f_{12}$

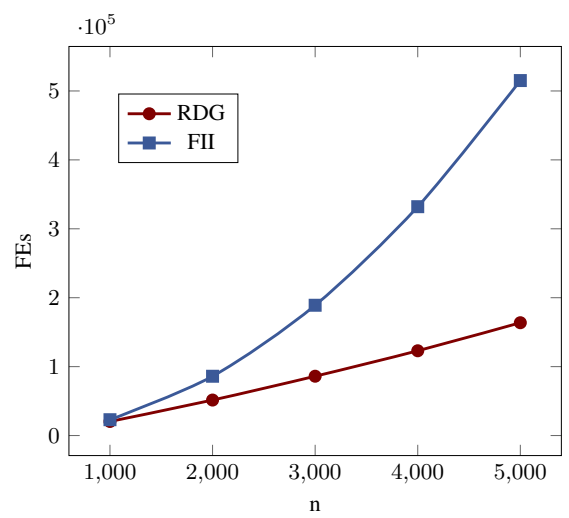

(b) CEC'2010 $f_{15}$

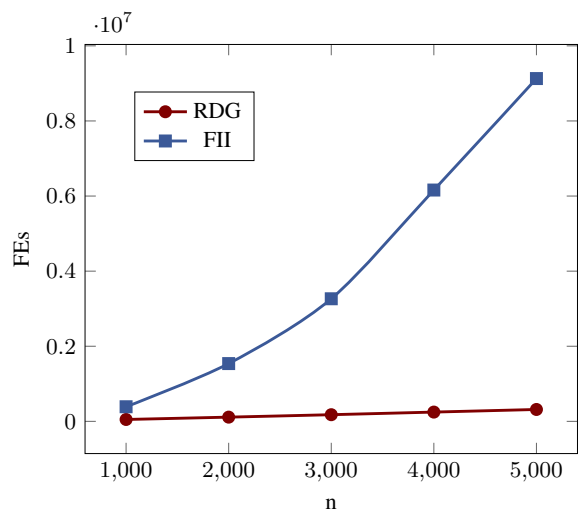

(C) CEC'2010 $f_{18}$

Fig. 4. The number of FEs used by RDG and FII methods when used to decompose the extended benchmark problems $\left(\right.$ CEC'2010 $f_{12}, f_{15}$ and $\left.f_{18}\right)$ with dimensionality equal to $1000,2000,3000,4000$, and 5000 .

TABLE III

THE EXTENDED CEC' $2010 f_{12}, f_{15}$ AND $f_{18}$ PROBLEMS. FOR EACH PROBLEM, THE NUMBER OF DECISION VARIABLES IN EACH NON-SEPARABLE SUB-COMPONENT IS FIXED TO 50, WHICH IS CONSISTENT WITH THE ORIGINAL BENCHMARK SET [20].

\begin{tabular}{cccc}
\hline Func & Dim & Sep Variables & Non-Sep Groups \\
\hline \multirow{4}{*}{$f_{12}$} & 1000 & 500 & 10 \\
& 2000 & 1000 & 20 \\
& 3000 & 1500 & 30 \\
& 4000 & 2000 & 40 \\
& 5000 & 2500 & 50 \\
\hline \multirow{5}{*}{$f_{15}, f_{18}$} & 1000 & 0 & 20 \\
& 2000 & 0 & 40 \\
& 3000 & 0 & 60 \\
& 4000 & 0 & 80 \\
\hline
\end{tabular}

variables. For example on CEC'2010 $f_{4}$, where there are 950 separable and 50 non-separable decision variables, the number of FEs used by FII $\left(3.69 \times 10^{3}\right)$ is slightly less than that used by RDG $\left(4.20 \times 10^{3}\right)$. However, on some benchmark problems especially those with conditional variable interactions e.g., CEC'2010 $f_{18}$ and $f_{20}$, RDG is much more efficient than FII, as shown in Fig. 3.

In fact, the number of FEs used by the FII method to decompose the CEC'2010 $f_{18}$ and $f_{20}$ problems is in $\Theta\left(n^{2}\right)$. It has been shown that FII uses $3 n+k n_{n}+k$ FEs when decomposing an $n$-dimensional problem with equally sized non-separable sub-components, where $n_{n}$ is the number of non-separable decision variables, and $k$ is the number of nonseparable sub-components [48]. As CEC'2010 $f_{18}$ and $f_{20}$ are Rosenbrock's functions, $n_{n}$ is equal to $n$ and each decision variable interacts with at most two other decision variables. Therefore the total number of FEs used by FII is around $3 n+n^{2} / 3+n / 3 \in \Theta\left(n^{2}\right)$.

On CEC'2010 $f_{6}$, the number of FEs used by RDG $(5.00 \times$ $\left.10^{4}\right)$ is greater than that used by FII $\left(3.05 \times 10^{3}\right)$. The reason for this is that the threshold estimated by the RDG method $\left(\epsilon=2.13 \times 10^{-5}\right)$ is too small, resulting in identifying some separable decision variables as non-separable. If RDG employs
TABLE IV

THE AVERAGE RANKING OF EACH DECOMPOSITION METHOD WHEN EMBEDDED INTO THE DECC OR CMAESCC FRAMEWORK TO SOLVE THE CEC'2010 AND CEC'2013 BENCHMARK PROBLEMS. THE RDG METHOD CONSISTENTLY ACHIEVES THE SMALLEST AVERAGE RANKING.

\begin{tabular}{ccccccccc}
\hline CCs & RDG & GDG & XDG & DG & DG2 & FII & D & RG \\
\hline DECC & 2.0 & 4.9 & 4.0 & 4.4 & 3.9 & 2.5 & 4.6 & 4.7 \\
CMAESCC & 1.7 & 3.1 & 3.3 & 4.0 & 2.9 & 2.2 & 6.7 & 5.7 \\
\hline
\end{tabular}

the same threshold with FII $(\epsilon=0.01)$, the FEs used by RDG will decrease to $5.06 \times 10^{3}$. However if FII employs the same threshold with RDG, the FEs used by FII will increase to $3.12 \times 10^{5}$.

To test the scalability of the RDG and FII methods, we extend some of the CEC'2010 benchmark problems from 1000 dimensions to 5000 dimensions (See Table III for details). When tested on the extended benchmark problems, we observe that the FEs used by the FII method increases more quickly than that used by the RDG method as the dimensionality increases, as shown in Fig. 4.

\section{B. Optimization Comparison}

The performances of the RDG, GDG, XDG, DG, DG2, FII, D (delta grouping), and RG methods when embedded in the DECC/CMAESCC framework to solve the CEC'2010 and CEC'2013 benchmark problems are presented in Fig. 5 and Fig. 6 respectively. On each benchmark problem, the eight methods are ranked from 1 to 8 (as labelled on the concentric circles in the radar charts) based on the results from 25 independent runs. The average ranking of each decomposition method across all the benchmark problems is presented in Table IV. The detailed optimization results from DECC-RDG and CMAESCC-RDG are presented in Table V and Table VI. The results from the other seven methods are placed in the supplementary documents due to page limits.

When embedded into the DECC/CMAESCC framework, the RDG method achieves the best solution quality when used to solve $16 / 15$ out of 23 partially separable benchmark 


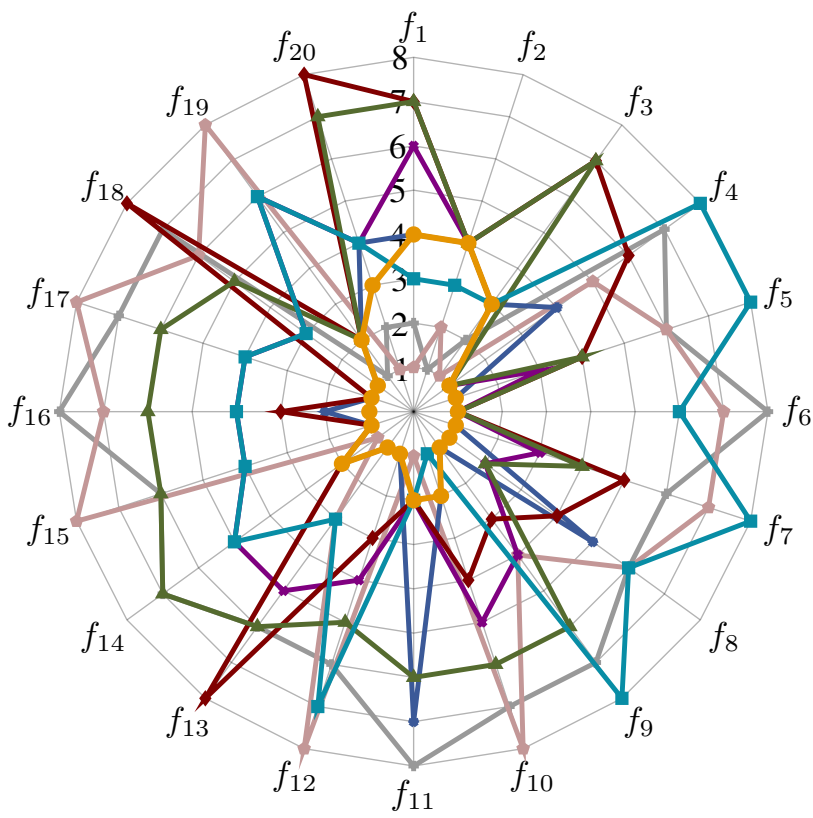

(a) CEC'2010

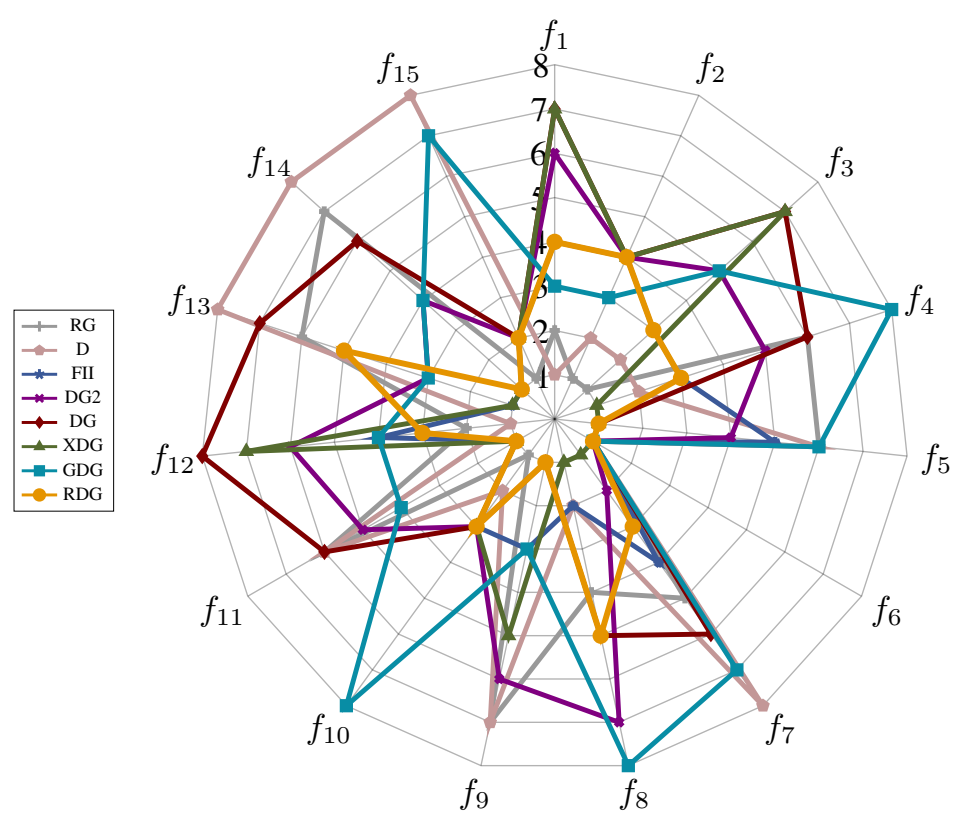

(b) CEC'2013

Fig. 5. The radar chart of the performance of RDG, GDG, XDG, DG, DG2, FII, D (delta grouping) and RG when embedded in the DECC framework to solve the CEC'2010 and CEC'2013 benchmark problems. On each benchmark problem, the eight methods are ranked from 1 to 8 (as labelled on the concentric circles) based on the results from 25 independent runs (Wilcoxon rank-sum tests $(\alpha=0.05)$ with Holm p-value correction).

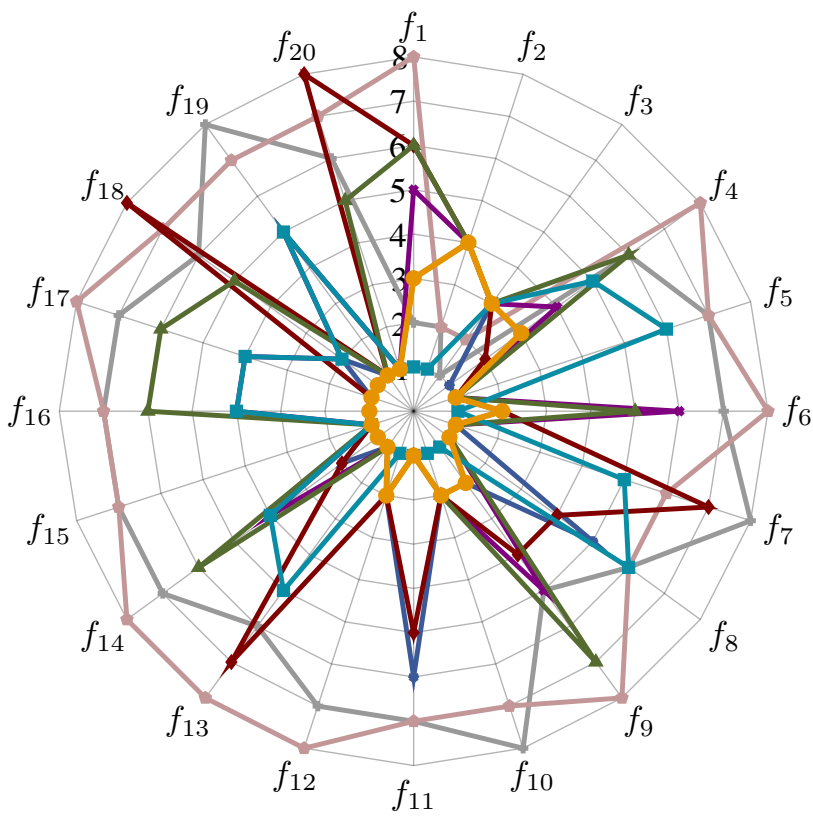

(a) CEC'2010

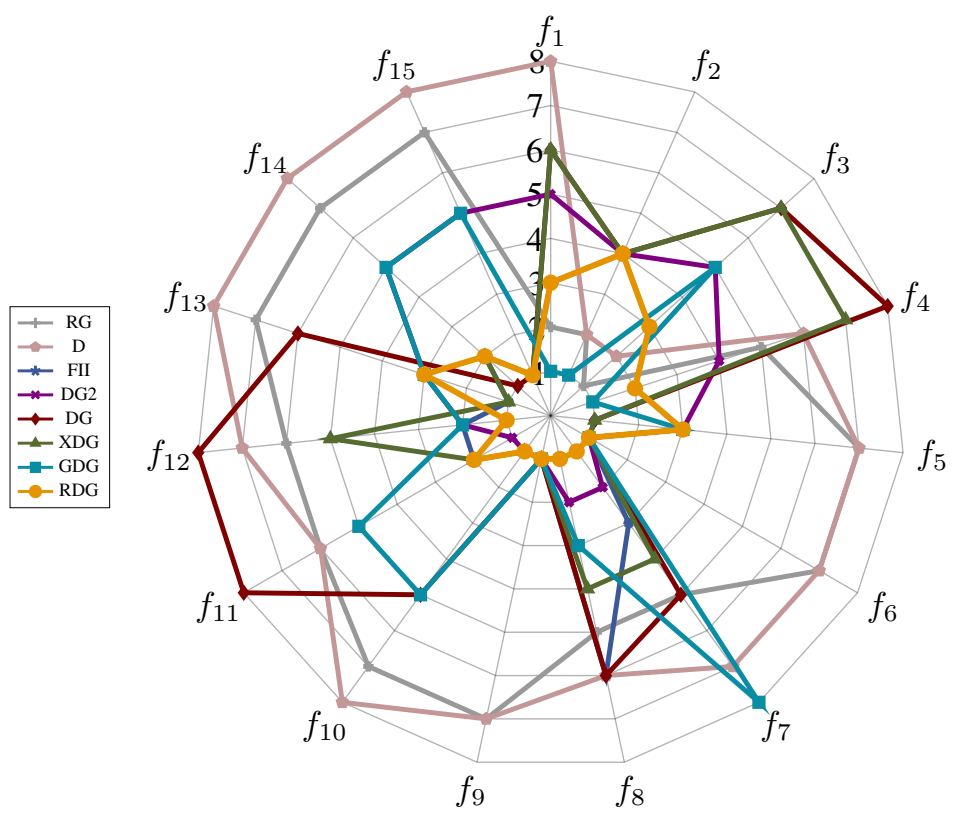

(b) CEC'2013

Fig. 6. The radar chart of the performance of RDG, GDG, XDG, DG, DG2, FII, D (delta grouping) and RG when embedded in the CMAESCC framework to solve the CEC'2010 and CEC'2013 benchmark problems. On each benchmark problem, the eight methods are ranked from 1 to 8 (as labelled on the concentric circles) based on the results from 25 independent runs (Wilcoxon rank-sum tests $(\alpha=0.05)$ with Holm p-value correction).

problems (CEC'2010 $f_{4^{-}} f_{18}$ and CEC'2013 $\left.f_{4^{-}} f_{11}\right)$. It obtains the smallest average ranking across all the benchmark problems investigated. The RDG method generally uses the smallest number of FEs in the decomposition stage, assigning more computational resources to optimize the problems, when compared against the other automatic decomposition methods.
On the fully separable and some fully non-separable problems, the RDG method is outperformed by the two manual decomposition methods - D (delta grouping) and RG, as RDG does not actually perform any decomposition for these problems. However, the performance of the D (delta grouping) and RG methods deteriorates quickly on the partially separable 


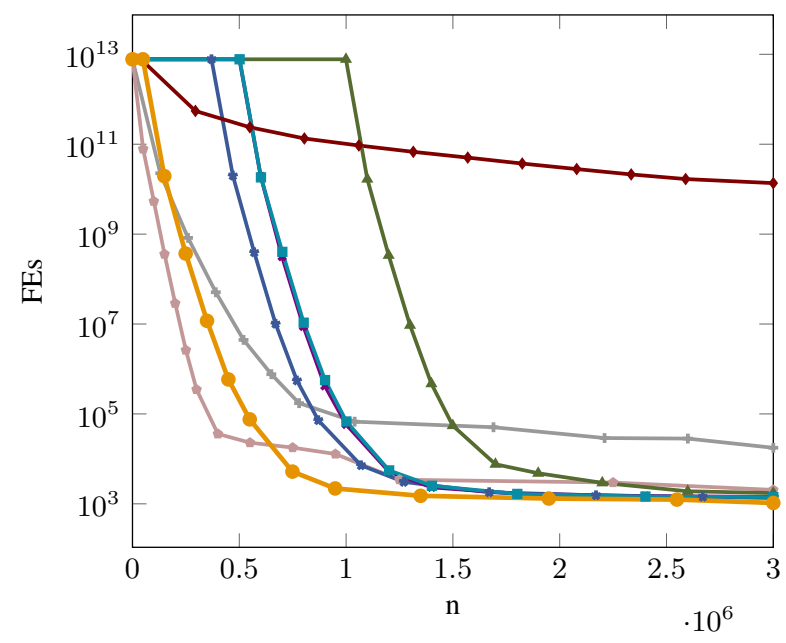

(a) DECC
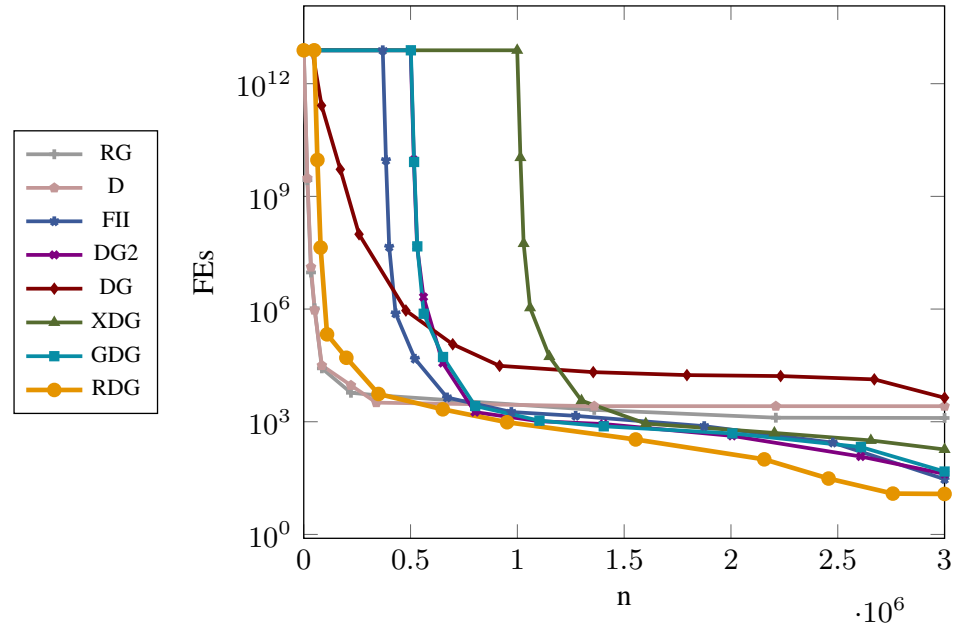

(b) CMAESCC

Fig. 7. The convergence curves of the RDG, GDG, XDG, DG, DG2, FII, D (delta grouping) and RG methods when embedded into the DECC and CMAESCC frameworks to solve the CEC'2010 $f_{18}$ problem. The horizontal axis represents the number of FEs used in the evolutionary process. The vertical axis represents the median of the best fitness found.

problems.

On some benchmark problems with separable decision variables, e.g., CEC'2010 $f_{10}$, the GDG method generates better solution quality than RDG. The reason for this is that GDG further divides the separable decision variables into several sub-components. Interestingly, the GDG method achieves the first place when embedded into CMAESCC, however the last place when embedded into DECC, to solve the CEC'2010 $f_{9}$.

The DG method performs poorly on overlapping benchmark problems (e.g., CEC'2010 $f_{8}, f_{13}, f_{18}$, and $f_{20}$ ). The reason for this is that the DG method can not completely identify interaction between decision variables in an overlapping problem. Once all the variable interactions are identified and all the linked decision variables are placed into one subcomponent, the solution quality can be greatly improved by several magnitudes.

The DG2 method generates the best solution quality when embedded into the CMAESCC framework to solve the CEC'2013 $f_{11}$ problem. The reason for this is that DG2 achieves $100 \%$ accuracy when used to decompose this problem, which is higher than RDG. However on the other benchmark problems, the RDG method generally obtains equally well or statistically better solution quality than DG2.

The FII method performs well across the benchmark problems investigated. It achieves the second place according to the average ranking. However, on the benchmark problems where the decomposition by FII is less efficient e.g., CEC'2010 $f_{8}$, the RDG method can generate significantly better solution quality than FII.

The DECC-D (with delta grouping) algorithm achieves much better results than the other DECC based algorithms when used to solve the CEC'2010 $f_{3}$ and $f_{11}$ benchmark problems. An interesting observation is that both $f_{3}$ and $f_{11}$ are Ackley's functions [21]. Moreover, the DECC-D (with delta grouping) algorithm also performs well when used to solve the other Ackley's functions: CEC'2010 $f_{6}, f_{16}$ and
CEC'2013 $f_{3}, f_{6}, f_{10}$. So far, we don't know the reason why the DECC-D (with delta grouping) algorithm performs well on the Ackley's functions.

The convergence curves of the eight decomposition methods when embedded into the DECC/CMAESCC framework to solve the CEC'2010 $f_{18}$ problem are shown in Fig. 7. The RDG method uses the smallest number of FEs in the decomposition stage, therefore can generate better solution quality when compared against the other automatic decomposition methods.

In the next phase of the experimental study, we compare the performance of DECC-RDG and CMAESCC-RDG against the performance of two state-of-the-art algorithms - MOS and MA-SW-Chains. The experimental results of each algorithm when used to solve the CEC'2010 and CEC'2013 benchmark problems are presented in Table $\mathrm{V}$ and Table VI respectively.

The CMAESCC-RDG algorithm achieves the best solution quality on 22 out of 35 benchmark problems when compared against DECC-RDG, MOS and MA-SW-Chains. It does not perform well on the fully separable problems (CEC'2010 $f_{1}$ to $f_{3}$ and CEC'2013 $f_{1}$ to $f_{3}$ ). However on partially separable and fully non-separable problems, it generally achieves comparable or statistically better solution quality than the other algorithms (e.g., on CEC'2010 $f_{4}$ to $f_{9}$ and $f_{11}$ to $f_{19}$ ).

The DECC-RDG algorithm achieves the best solution quality when used to solve the CEC'2010 $f_{16}$ problem. On the other 34 benchmark problems, the DECC-RDG is outperformed by the CMAESCC-RDG. It may indicate that the subcomponent optimizer used by the CMAESCC framework is more effective than that used by the DECC framework.

The MOS algorithm achieves the best results when used to solve the fully separable problems (CEC'2010 $f_{1}-f_{3}$, and CEC'2013 $\left.f_{1}-f_{3}\right)$. However, on partially separable problems and fully non-separable problems, it is generally outperformed by the CMAESCC-RDG algorithm.

The MA-SW-Chains algorithm performs well on the CEC'2013 benchmark problems. It achieves the best results 
TABLE V

THE RESULTS OF THE DECC-RDG, CMAESCC-RDG, MOS AND MA-SW-CHAINS ALGORITHMS WHEN USED TO SOLVE THE CEC'2010 BENCHMARK PROBLEMS. THE BEST PERFORMANCES ARE HIGHLIGHTED IN BOLD (WILCOXON RANK-SUM TESTS $(\alpha=0.05)$ WITH HOLM P-VALUE CORRECTION).

\begin{tabular}{|c|c|c|c|c|c|}
\hline Func & Stats & DECC-RDG & CMAESCC-RDG & MOS & MA-SW-Chains \\
\hline \multirow{3}{*}{$f_{1}$} & Median & $1.50 \mathrm{e}-01$ & $2.86 \mathrm{e}+05$ & $0.00 \mathrm{e}+00$ & $2.67 \mathrm{e}-14$ \\
\hline & Mean & $2.07 \mathrm{e}+00$ & $2.84 \mathrm{e}+05$ & $1.50 \mathrm{e}-28$ & $3.80 \mathrm{e}-14$ \\
\hline & Std & $6.75 e+00$ & $2.28 \mathrm{e}+04$ & $5.55 \mathrm{e}-28$ & $4.91 \mathrm{e}-14$ \\
\hline \multirow{3}{*}{$f_{2}$} & Median & $4.35 e+03$ & $4.43 e+03$ & $0.00 \mathrm{e}+00$ & $8.47 \mathrm{e}+02$ \\
\hline & Mean & $4.38 \mathrm{e}+03$ & $4.42 \mathrm{e}+03$ & $0.00 \mathrm{e}+00$ & $8.40 \mathrm{e}+02$ \\
\hline & Std & $1.72 \mathrm{e}+02$ & $1.76 \mathrm{e}+02$ & $0.00 \mathrm{e}+00$ & $4.88 \mathrm{e}+01$ \\
\hline \multirow{3}{*}{$f_{3}$} & Median & $1.65 \mathrm{e}+01$ & $1.12 \mathrm{e}+00$ & $0.00 \mathrm{e}+00$ & $5.16 \mathrm{e}-13$ \\
\hline & Mean & $1.65 \mathrm{e}+01$ & $1.05 \mathrm{e}+00$ & $0.00 \mathrm{e}+00$ & $5.76 \mathrm{e}-13$ \\
\hline & Std & $3.35 \mathrm{e}-01$ & $3.49 \mathrm{e}-01$ & $0.00 \mathrm{e}+00$ & $2.73 \mathrm{e}-13$ \\
\hline \multirow{3}{*}{$f_{4}$} & Median & $5.75 \mathrm{e}+11$ & $9.97 \mathrm{e}+05$ & $4.94 \mathrm{e}+11$ & $3.10 \mathrm{e}+11$ \\
\hline & Mean & $6.68 \mathrm{e}+11$ & $1.01 \mathrm{e}+06$ & $5.16 \mathrm{e}+11$ & $2.97 \mathrm{e}+11$ \\
\hline & Std & $3.33 \mathrm{e}+11$ & $9.37 e+04$ & $1.85 \mathrm{e}+11$ & $6.19 \mathrm{e}+10$ \\
\hline \multirow{3}{*}{$f_{5}$} & Median & $1.31 \mathrm{e}+08$ & $9.05 e+07$ & $5.00 \mathrm{e}+08$ & $2.30 \mathrm{e}+08$ \\
\hline & Mean & $1.28 \mathrm{e}+08$ & $9.52 \mathrm{e}+07$ & $4.93 \mathrm{e}+08$ & $2.18 \mathrm{e}+08$ \\
\hline & Std & $1.92 \mathrm{e}+07$ & $2.22 \mathrm{e}+07$ & $6.93 \mathrm{e}+07$ & $5.75 e+07$ \\
\hline \multirow{3}{*}{$f_{6}$} & Median & $1.61 \mathrm{e}+01$ & $1.04 \mathrm{e}+00$ & $1.97 \mathrm{e}+07$ & $2.45 e+00$ \\
\hline & Mean & $1.61 \mathrm{e}+01$ & 9.17e-01 & $1.97 \mathrm{e}+07$ & $1.42 \mathrm{e}+05$ \\
\hline & Std & $3.64 \mathrm{e}-01$ & $4.23 \mathrm{e}-01$ & $1.15 \mathrm{e}+05$ & $3.96 \mathrm{e}+05$ \\
\hline \multirow{3}{*}{$f_{7}$} & Median & $2.46 \mathrm{e}+00$ & $7.41 \mathrm{e}-19$ & $2.27 \mathrm{e}+07$ & $7.94 \mathrm{e}-03$ \\
\hline & Mean & $2.16 \mathrm{e}+01$ & $7.41 \mathrm{e}-19$ & $3.54 \mathrm{e}+07$ & $1.17 \mathrm{e}+02$ \\
\hline & Std & $7.56 \mathrm{e}+01$ & $8.35 \mathrm{e}-20$ & $3.22 \mathrm{e}+07$ & $2.37 \mathrm{e}+02$ \\
\hline \multirow{3}{*}{$f_{8}$} & Median & $3.66 \mathrm{e}+00$ & $1.83 \mathrm{e}-17$ & $2.14 \mathrm{e}+06$ & $2.76 e+06$ \\
\hline & Mean & $1.59 \mathrm{e}+05$ & $6.37 \mathrm{e}+05$ & $3.75 e+06$ & $6.90 \mathrm{e}+06$ \\
\hline & Std & $7.97 \mathrm{e}+05$ & $1.49 \mathrm{e}+06$ & $4.40 \mathrm{e}+06$ & $1.90 \mathrm{e}+07$ \\
\hline \multirow{3}{*}{$f_{9}$} & Median & $4.65 e+07$ & $4.80 \mathrm{e}+06$ & $1.18 \mathrm{e}+07$ & $1.48 \mathrm{e}+07$ \\
\hline & Mean & $4.69 \mathrm{e}+07$ & $4.82 \mathrm{e}+06$ & $1.13 \mathrm{e}+07$ & $1.49 \mathrm{e}+07$ \\
\hline & Std & $5.21 \mathrm{e}+06$ & $3.88 \mathrm{e}+05$ & $1.61 \mathrm{e}+06$ & $1.61 \mathrm{e}+06$ \\
\hline \multirow{3}{*}{$f_{10}$} & Median & $4.33 \mathrm{e}+03$ & $2.78 \mathrm{e}+03$ & $6.35 e+03$ & $2.02 \mathrm{e}+03$ \\
\hline & Mean & $4.33 e+03$ & $2.79 \mathrm{e}+03$ & $6.28 \mathrm{e}+03$ & $2.01 \mathrm{e}+03$ \\
\hline & Std & $1.39 \mathrm{e}+02$ & $1.17 \mathrm{e}+02$ & $3.12 \mathrm{e}+02$ & $1.59 \mathrm{e}+02$ \\
\hline \multirow{3}{*}{$f_{11}$} & Median & $1.03 \mathrm{e}+01$ & $1.51 \mathrm{e}-12$ & $2.84 \mathrm{e}+01$ & $3.77 \mathrm{e}+01$ \\
\hline & Mean & $1.03 \mathrm{e}+01$ & $3.58 \mathrm{e}-02$ & $3.08 \mathrm{e}+01$ & $3.86 \mathrm{e}+01$ \\
\hline & Std & $8.50 \mathrm{e}-01$ & $1.79 \mathrm{e}-01$ & $6.07 \mathrm{e}+00$ & $8.06 \mathrm{e}+00$ \\
\hline \multirow{3}{*}{$f_{12}$} & Median & $1.38 \mathrm{e}+03$ & $4.30 \mathrm{e}-22$ & $3.46 e+03$ & $3.09 \mathrm{e}-06$ \\
\hline & Mean & $1.53 \mathrm{e}+03$ & $4.22 \mathrm{e}-22$ & $4.39 e+03$ & $3.24 \mathrm{e}-06$ \\
\hline & Std & $4.66 \mathrm{e}+02$ & $8.38 \mathrm{e}-23$ & $2.92 \mathrm{e}+03$ & $5.78 \mathrm{e}-07$ \\
\hline \multirow{3}{*}{$f_{13}$} & Median & $6.12 \mathrm{e}+02$ & $3.98 \mathrm{e}+00$ & $3.19 \mathrm{e}+02$ & $8.61 \mathrm{e}+02$ \\
\hline & Mean & $7.12 \mathrm{e}+02$ & $4.78 \mathrm{e}+00$ & $3.32 \mathrm{e}+02$ & $9.83 e+02$ \\
\hline & Std & $2.52 \mathrm{e}+02$ & $3.98 \mathrm{e}+00$ & $1.19 \mathrm{e}+02$ & $5.66 \mathrm{e}+02$ \\
\hline \multirow{3}{*}{$f_{14}$} & Median & $3.47 \mathrm{e}+08$ & 3.90e-20 & $2.04 \mathrm{e}+07$ & $3.23 e+07$ \\
\hline & Mean & $3.47 \mathrm{e}+08$ & 3.91e-20 & $2.05 e+07$ & $3.25 \mathrm{e}+07$ \\
\hline & Std & $2.31 \mathrm{e}+07$ & 2.11e-20 & $3.60 \mathrm{e}+06$ & $2.46 \mathrm{e}+06$ \\
\hline \multirow{3}{*}{$f_{15}$} & Median & $5.82 \mathrm{e}+03$ & $1.92 \mathrm{e}+03$ & $1.29 \mathrm{e}+04$ & $2.67 \mathrm{e}+03$ \\
\hline & Mean & $5.84 \mathrm{e}+03$ & $1.94 \mathrm{e}+03$ & $1.29 \mathrm{e}+04$ & $2.68 \mathrm{e}+03$ \\
\hline & Std & $1.01 \mathrm{e}+02$ & $1.10 \mathrm{e}+02$ & $3.48 \mathrm{e}+02$ & $9.95 \mathrm{e}+01$ \\
\hline \multirow{3}{*}{$f_{16}$} & Median & $2.66 \mathrm{e}-13$ & $8.41 \mathrm{e}-13$ & $3.97 \mathrm{e}+02$ & $9.32 \mathrm{e}+01$ \\
\hline & Mean & $2.67 \mathrm{e}-13$ & $8.43 e-13$ & $3.96 \mathrm{e}+02$ & $9.95 \mathrm{e}+01$ \\
\hline & Std & $9.81 \mathrm{e}-15$ & $2.10 \mathrm{e}-14$ & $3.47 \mathrm{e}+00$ & $1.53 \mathrm{e}+01$ \\
\hline & Median & $4.08 \mathrm{e}+04$ & $6.89 \mathrm{e}-24$ & $7.30 \mathrm{e}+03$ & $1.28 \mathrm{e}+00$ \\
\hline$f_{17}$ & Mean & $4.07 e+04$ & $6.90 \mathrm{e}-24$ & $8.45 e+03$ & $1.27 \mathrm{e}+00$ \\
\hline & Std & $2.55 \mathrm{e}+03$ & $2.05 \mathrm{e}-25$ & $5.04 \mathrm{e}+03$ & $1.24 \mathrm{e}-01$ \\
\hline & Median & $1.19 \mathrm{e}+03$ & $1.55 \mathrm{e}+01$ & $7.78 \mathrm{e}+02$ & $1.41 \mathrm{e}+03$ \\
\hline$f_{18}$ & Mean & $1.20 \mathrm{e}+03$ & $1.50 \mathrm{e}+01$ & $8.96 \mathrm{e}+02$ & $1.57 \mathrm{e}+03$ \\
\hline & Std & $1.07 \mathrm{e}+02$ & $7.19 \mathrm{e}+00$ & $4.03 e+02$ & $6.73 e+02$ \\
\hline & Median & $1.71 \mathrm{e}+06$ & $5.63 \mathrm{e}+03$ & $5.71 \mathrm{e}+05$ & $3.75 e+05$ \\
\hline$f_{19}$ & Mean & $1.71 \mathrm{e}+06$ & $5.46 \mathrm{e}+03$ & $5.49 \mathrm{e}+05$ & $3.80 \mathrm{e}+05$ \\
\hline & Std & $8.91 \mathrm{e}+04$ & $7.07 e+02$ & $8.38 \mathrm{e}+04$ & $2.34 \mathrm{e}+04$ \\
\hline & Median & $3.70 \mathrm{e}+03$ & $8.55 e+02$ & $7.40 \mathrm{e}+01$ & $1.04 \mathrm{e}+03$ \\
\hline$f_{20}$ & Mean & $6.96 \mathrm{e}+03$ & $8.26 \mathrm{e}+02$ & $9.23 e+01$ & $1.06 e+03$ \\
\hline & Std & $1.27 \mathrm{e}+04$ & $6.35 \mathrm{e}+01$ & $8.99 \mathrm{e}+01$ & $9.38 \mathrm{e}+01$ \\
\hline
\end{tabular}

TABLE VI

THE RESUlTS OF THE DECC-RDG, CMAESCC-RDG, MOS AND MA-SW-CHAINS ALGORITHMS WHEN USED TO SOLVE THE CEC'2013 BENCHMARK PROBLEMS. THE BEST PERFORMANCES ARE HIGHLIGHTED IN BOLD (WILCOXON RANK-SUM TESTS $(\alpha=0.05)$ WITH HOLM P-VALUE CORRECTION).

\begin{tabular}{|c|c|c|c|c|c|}
\hline Func & Stats & DECC-RDG & CMAESCC-RDG & MOS & MA-SW-Chains \\
\hline \multirow{3}{*}{$f_{1}$} & Median & $5.32 \mathrm{e}-01$ & $2.84 \mathrm{e}+05$ & $1.34 \mathrm{e}-30$ & $7.12 \mathrm{e}-13$ \\
\hline & Mean & $3.73 \mathrm{e}+01$ & $2.89 \mathrm{e}+05$ & 3.10e-29 & $1.34 \mathrm{e}-12$ \\
\hline & Std & $1.24 \mathrm{e}+02$ & $3.27 \mathrm{e}+04$ & $4.53 \mathrm{e}-29$ & $2.45 \mathrm{e}-12$ \\
\hline \multirow{3}{*}{$f_{2}$} & Median & $1.29 \mathrm{e}+04$ & $4.66 \mathrm{e}+03$ & $1.90 \mathrm{e}+01$ & $1.24 \mathrm{e}+03$ \\
\hline & Mean & $1.27 \mathrm{e}+04$ & $4.68 \mathrm{e}+03$ & $1.83 e+01$ & $1.25 \mathrm{e}+03$ \\
\hline & Std & $6.40 \mathrm{e}+02$ & $1.77 \mathrm{e}+02$ & $4.65 \mathrm{e}+00$ & $1.05 e+02$ \\
\hline \multirow{3}{*}{$f_{3}$} & Median & $2.13 \mathrm{e}+01$ & $2.03 \mathrm{e}+01$ & $1.49 \mathrm{e}-13$ & $6.83 \mathrm{e}-13$ \\
\hline & Mean & $2.13 \mathrm{e}+01$ & $2.03 \mathrm{e}+01$ & $1.65 \mathrm{e}-13$ & $6.85 e-13$ \\
\hline & Std & $1.64 \mathrm{e}-02$ & $4.96 \mathrm{e}-02$ & $1.02 \mathrm{e}-13$ & $2.12 \mathrm{e}-13$ \\
\hline \multirow{3}{*}{$f_{4}$} & Median & $4.01 \mathrm{e}+10$ & $5.83 \mathrm{e}+06$ & $1.23 e+10$ & $2.75 e+09$ \\
\hline & Mean & $4.44 \mathrm{e}+10$ & $5.90 \mathrm{e}+06$ & $1.40 \mathrm{e}+10$ & $3.81 \mathrm{e}+09$ \\
\hline & Std & $1.77 \mathrm{e}+10$ & $6.56 \mathrm{e}+05$ & $7.65 e+09$ & $2.73 e+09$ \\
\hline \multirow{3}{*}{$f_{5}$} & Median & $5.09 \mathrm{e}+06$ & $2.19 \mathrm{e}+06$ & $1.12 \mathrm{e}+07$ & $2.03 e+06$ \\
\hline & Mean & $5.09 \mathrm{e}+06$ & $2.20 \mathrm{e}+06$ & $1.15 \mathrm{e}+07$ & $2.25 \mathrm{e}+06$ \\
\hline & Std & $4.81 \mathrm{e}+05$ & $3.76 \mathrm{e}+05$ & $1.82 \mathrm{e}+06$ & $1.30 \mathrm{e}+06$ \\
\hline \multirow{3}{*}{$f_{6}$} & Median & $1.06 \mathrm{e}+06$ & $9.95 \mathrm{e}+05$ & $9.78 \mathrm{e}+05$ & $6.33 \mathrm{e}+02$ \\
\hline & Mean & $1.06 \mathrm{e}+06$ & $9.95 \mathrm{e}+05$ & $9.83 e+05$ & $1.86 e+04$ \\
\hline & Std & $1.21 \mathrm{e}+03$ & $2.88 \mathrm{e}+01$ & $8.22 \mathrm{e}+03$ & $2.54 \mathrm{e}+04$ \\
\hline \multirow{3}{*}{$f_{7}$} & Median & $5.41 \mathrm{e}+07$ & $2.94 \mathrm{e}-20$ & $1.30 \mathrm{e}+07$ & $4.03 e+06$ \\
\hline & Mean & $6.42 \mathrm{e}+07$ & $8.12 \mathrm{e}-17$ & $2.33+07$ & $3.85 \mathrm{e}+06$ \\
\hline & Std & $2.97 \mathrm{e}+07$ & $2.17 e-16$ & $3.62 \mathrm{e}+07$ & $6.34 \mathrm{e}+05$ \\
\hline \multirow{3}{*}{$f_{8}$} & Median & $4.74 \mathrm{e}+15$ & $8.71 \mathrm{e}+06$ & $1.15 \mathrm{e}+15$ & $4.60 \mathrm{e}+13$ \\
\hline & Mean & $5.04 \mathrm{e}+15$ & $9.74 \mathrm{e}+06$ & $1.65 e+15$ & $4.62 \mathrm{e}+13$ \\
\hline & Std & $1.86 \mathrm{e}+15$ & $5.83 \mathrm{e}+06$ & $1.76 \mathrm{e}+15$ & $9.02 \mathrm{e}+12$ \\
\hline \multirow{3}{*}{$f_{9}$} & Median & $4.85 \mathrm{e}+08$ & $1.57 \mathrm{e}+08$ & $9.08 \mathrm{e}+08$ & $1.42 \mathrm{e}+08$ \\
\hline & Mean & $4.82 \mathrm{e}+08$ & $1.65 \mathrm{e}+08$ & $9.02 \mathrm{e}+08$ & $1.44 \mathrm{e}+08$ \\
\hline & Std & $3.06 \mathrm{e}+07$ & $4.16 \mathrm{e}+07$ & $1.04 \mathrm{e}+08$ & $1.55 \mathrm{e}+07$ \\
\hline \multirow{3}{*}{$f_{10}$} & Median & $9.44 \mathrm{e}+07$ & $9.04 \mathrm{e}+07$ & $8.82 \mathrm{e}+07$ & $3.34 \mathrm{e}+02$ \\
\hline & Mean & $9.44 \mathrm{e}+07$ & $9.12 \mathrm{e}+07$ & $6.66 \mathrm{e}+07$ & $3.72 e+04$ \\
\hline & Std & $2.06 \mathrm{e}+05$ & $1.53 \mathrm{e}+06$ & $3.01 \mathrm{e}+07$ & $6.25 \mathrm{e}+04$ \\
\hline \multirow{3}{*}{$f_{11}$} & Median & $5.31 \mathrm{e}+08$ & $1.64 \mathrm{e}+07$ & $1.82 \mathrm{e}+09$ & $2.10 \mathrm{e}+08$ \\
\hline & Mean & $5.38 \mathrm{e}+08$ & $1.62 \mathrm{e}+07$ & $3.87 \mathrm{e}+10$ & $2.10 \mathrm{e}+08$ \\
\hline & Std & $1.34 \mathrm{e}+08$ & $6.11 \mathrm{e}+05$ & $1.06 e+11$ & $2.35 \mathrm{e}+07$ \\
\hline \multirow{3}{*}{$f_{12}$} & Median & $3.77 \mathrm{e}+03$ & $1.01 \mathrm{e}+03$ & $6.89 \mathrm{e}+01$ & $1.25 \mathrm{e}+03$ \\
\hline & Mean & $4.85 \mathrm{e}+03$ & $9.81 \mathrm{e}+02$ & $8.64 \mathrm{e}+01$ & $1.24 \mathrm{e}+03$ \\
\hline & Std & $3.06 \mathrm{e}+03$ & $7.30 \mathrm{e}+01$ & $7.82 \mathrm{e}+01$ & $8.33 \mathrm{e}+01$ \\
\hline \multirow{3}{*}{$f_{13}$} & Median & $3.16 \mathrm{e}+09$ & $2.49 \mathrm{e}+06$ & $8.45 e+08$ & $1.91 \mathrm{e}+07$ \\
\hline & Mean & $3.06 \mathrm{e}+09$ & $2.47 \mathrm{e}+06$ & $1.09 \mathrm{e}+09$ & $3.58 \mathrm{e}+07$ \\
\hline & Std & $6.68 \mathrm{e}+08$ & $3.83 \mathrm{e}+05$ & $7.69 e+08$ & $4.30 \mathrm{e}+07$ \\
\hline \multirow{3}{*}{$f_{14}$} & Median & $2.50 \mathrm{e}+09$ & $2.74 \mathrm{e}+07$ & $2.27 \mathrm{e}+09$ & $1.43 \mathrm{e}+08$ \\
\hline & Mean & $2.87 \mathrm{e}+09$ & $2.76 \mathrm{e}+07$ & $6.65 e+09$ & $1.45 \mathrm{e}+08$ \\
\hline & Std & $1.73 \mathrm{e}+09$ & $1.49 \mathrm{e}+06$ & $1.62 \mathrm{e}+10$ & $1.60 \mathrm{e}+07$ \\
\hline \multirow{3}{*}{$f_{15}$} & Median & $9.67 \mathrm{e}+06$ & $2.18 \mathrm{e}+06$ & $1.24 \mathrm{e}+08$ & $5.80 \mathrm{e}+06$ \\
\hline & Mean & $9.75 e+06$ & $2.19 \mathrm{e}+06$ & $1.33 \mathrm{e}+08$ & $5.98 \mathrm{e}+06$ \\
\hline & Std & $1.91 \mathrm{e}+06$ & $2.28 \mathrm{e}+05$ & $6.05 e+07$ & $1.42 \mathrm{e}+06$ \\
\hline
\end{tabular}

when used to solve the CEC'2013 $f_{5}, f_{6}, f_{9}$, and $f_{10}$ problems. However, on the other partially separable and fully nonseparable benchmark problems, it is consistently outperformed by the CMAESCC-RDG algorithm. In some cases, the best solution found by the CMAESCC-RDG algorithm is much better than that found by the MA-SW-Chains algorithm. 


\section{CONCLUSION}

In this paper, we have investigated the influence of problem decomposition on the performance of $\mathrm{CC}$ algorithms when used to solve LSGO problems. A robust decomposition method - RDG - was proposed, which can decompose an $n$-dimensional problem using $\mathcal{O}(n \log (n))$ FEs based on a measure of non-linearity between decision variables. Significantly, RDG outperformed seven other decomposition methods when embedded into the DECC/CMAESCC framework and tested across a suite of benchmark LSGO problems. When compared against two other state-of-the-art hybrid algorithms, the CMAESCC-RDG algorithm achieved statistically significantly better results.

In future work, we plan to apply the CMAESCC-RDG algorithm to solve real-world LSGO problems. Another direction worth pursuing is focused on the modification of the RDG method to the combinatorial and multi-objective spaces.

\section{ACKNOWLEDGMENT}

The authors would like to thank Mario A. Muñoz, Wei Wang for their valuable comments.

\section{REFERENCES}

[1] P. Benner, "Solving large-scale control problems," Control Systems, IEEE, vol. 24, no. 1, pp. 44-59, 2004.

[2] S. Shan and G. G. Wang, "Survey of modeling and optimization strategies to solve high-dimensional design problems with computationallyexpensive black-box functions," Structural and Multidisciplinary Optimization, vol. 41, no. 2, pp. 219-241, 2010.

[3] H. Liu and L. Yu, "Toward integrating feature selection algorithms for classification and clustering," Knowledge and Data Engineering, IEEE Transactions on, vol. 17, no. 4, pp. 491-502, 2005.

[4] M. N. Omidvar, X. Li, and K. Tang, "Designing benchmark problems for large-scale continuous optimization," Information Sciences, vol. 316, pp. 419-436, 2015.

[5] T. Weise, R. Chiong, and K. Tang, "Evolutionary optimization: Pitfalls and booby traps," Journal of Computer Science and Technology, vol. 27, no. 5, pp. 907-936, 2012

[6] W. Dong, T. Chen, P. Tino, and X. Yao, "Scaling up estimation of distribution algorithms for continuous optimization," Evolutionary Computation, IEEE Transactions on, vol. 17, no. 6, pp. 797-822, 2013.

[7] S. Mahdavi, M. E. Shiri, and S. Rahnamayan, "Metaheuristics in largescale global continues optimization: A survey," Information Sciences, vol. 295, pp. 407-428, 2015.

[8] A. LaTorre, S. Muelas, and J.-M. Peña, "A comprehensive comparison of large scale global optimizers," Information Sciences, vol. 316, pp. 517-549, 2015.

[9] M. A. Potter and K. A. De Jong, "A cooperative coevolutionary approach to function optimization," in Parallel problem solving from nature PPSN III. Springer, 1994, pp. 249-257.

[10] M. N. Omidvar, X. Li, Y. Mei, and X. Yao, "Cooperative co-evolution with differential grouping for large scale optimization," Evolutionary Computation, IEEE Transactions on, vol. 18, no. 3, pp. 378-393, 2014.

[11] Y. Mei, X. Li, and X. Yao, "Cooperative coevolution with route distance grouping for large-scale capacitated arc routing problems," Evolutionary Computation, IEEE Transactions on, vol. 18, no. 3, pp. 435-449, 2014

[12] E. Sayed, D. Essam, R. Sarker, and S. Elsayed, "Decompositionbased evolutionary algorithm for large scale constrained problems," Information Sciences, vol. 316, pp. 457-486, 2015.

[13] K. C. Tan, Y. Yang, and C. K. Goh, "A distributed cooperative coevolutionary algorithm for multiobjective optimization," Evolutionary Computation, IEEE Transactions on, vol. 10, no. 5, pp. 527-549, 2006.

[14] C. Goh and K. C. Tan, "A competitive-cooperative coevolutionary paradigm for dynamic multiobjective optimization," Evolutionary Computation, IEEE Transactions on, vol. 13, no. 1, pp. 103-127, 2009.

[15] Y. Sun, M. Kirley, and S. K. Halgamuge, "Extended differential grouping for large scale global optimization with direct and indirect variable interactions," in Proceedings of the 2015 on Genetic and Evolutionary Computation Conference. ACM, 2015, pp. 313-320.
[16] W. Chen and K. Tang, "Impact of problem decomposition on cooperative coevolution," in 2013 IEEE Congress on Evolutionary Computation. IEEE, 2013, pp. 733-740.

[17] H. Liu, Y. Wang, X. Liu, and S. Guan, "Empirical study of effect of grouping strategies for large scale optimization," in Neural Networks (IJCNN), 2016 International Joint Conference on. IEEE, 2016, pp. 3433-3439.

[18] B. Kazimipour, M. N. Omidvar, X. Li, and A. Qin, "A sensitivity analysis of contribution-based cooperative co-evolutionary algorithms," in 2015 IEEE Congress on Evolutionary Computation (CEC). IEEE, 2015, pp. 417-424.

[19] Z. Yang, K. Tang, and X. Yao, "Large scale evolutionary optimization using cooperative coevolution," Information Sciences, vol. 178, no. 15, pp. 2985-2999, 2008 .

[20] K. Tang, X. Yao, and P. Suganthan, "Benchmark functions for the CEC'2010 special session and competition on large scale global optimization," Technique Report, USTC, Natrue Inspired Computation and Applications Laboratory, no. 1, pp. 1-23, 2010.

[21] X. Li, K. Tang, M. N. Omidvar, Z. Yang, and K. Qin, "Benchmark functions for the CEC 2013 special session and competition on largescale global optimization," gene, vol. 7, no. 33, p. 8, 2013.

[22] R. Tang, Z. Wu, and Y. Fang, "Adaptive multi-context cooperatively coevolving particle swarm optimization for large-scale problems," Soft Computing, pp. 1-20, 2016.

[23] M. Yang, M. N. Omidvar, C. Li, X. Li, Z. Cai, B. Kazimipour, and $\mathrm{X}$. Yao, "Efficient resource allocation in cooperative co-evolution for large-scale global optimization," IEEE Transactions on Evolutionary Computation, 2016.

[24] A. Kabán, J. Bootkrajang, and R. J. Durrant, "Toward Large-Scale Continuous EDA: A Random Matrix Theory Perspective," Evolutionary computation, 2015.

[25] J. Brest and M. S. Maučec, "Self-adaptive differential evolution algorithm using population size reduction and three strategies," Soft Computing, vol. 15, no. 11, pp. 2157-2174, 2011.

[26] H. Wang, Z. Wu, and S. Rahnamayan, "Enhanced opposition-based differential evolution for solving high-dimensional continuous optimization problems," Soft Computing, vol. 15, no. 11, pp. 2127-2140, 2011.

[27] R. Cheng and Y. Jin, "A social learning particle swarm optimization algorithm for scalable optimization," Information Sciences, vol. 291, pp. 43-60, 2015.

[28] R. Cheng and Y. Jin, "A competitive swarm optimizer for large scale optimization," Cybernetics, IEEE Transactions on, vol. 45, no. 2, pp. 191-204, 2015.

[29] L. Tseng and C. Chen, "Multiple trajectory search for large scale global optimization," in Evolutionary Computation, 2008. CEC 2008.(IEEE World Congress on Computational Intelligence). IEEE Congress on. IEEE, 2008, pp. 3052-3059.

[30] A. LaTorre, S. Muelas, and J.-M. Peña, "A MOS-based dynamic memetic differential evolution algorithm for continuous optimization: a scalability test," Soft Computing, vol. 15, no. 11, pp. 2187-2199, 2011.

[31] Y. Sun, M. Kirley, and S. K. Halgamuge, "Quantifying variable interactions in Continuous Optimization Problems," Evolutionary Computation, IEEE Transaction on, In Press, DOI: 10.1109/TEVC.2016.2599164.

[32] F. Van den Bergh and A. P. Engelbrecht, "A cooperative approach to particle swarm optimization," Evolutionary Computation, IEEE Transactions on, vol. 8, no. 3, pp. 225-239, 2004.

[33] X. Zheng, D. Lu, X. Wang, and H. Liu, "A cooperative coevolutionary biogeography-based optimizer," Applied Intelligence, vol. 43, no. 1, pp. 95-111, 2015.

[34] X. Li and X. Yao, "Cooperatively coevolving particle swarms for large scale optimization," Evolutionary Computation, IEEE Transactions on, vol. 16, no. 2, pp. 210-224, 2012.

[35] Y. Ren and Y. Wu, "An efficient algorithm for high-dimensional function optimization," Soft Computing, vol. 17, no. 6, pp. 995-1004, 2013.

[36] M. N. Omidvar, X. Li, Z. Yang, and X. Yao, "Cooperative co-evolution for large scale optimization through more frequent random grouping," in Evolutionary Computation (CEC), 2010 IEEE Congress on. IEEE, 2010, pp. 1-8.

[37] Z. Yang, K. Tang, and X. Yao, "Multilevel cooperative coevolution for large scale optimization," in Evolutionary Computation, 2008. CEC 2008.(IEEE World Congress on Computational Intelligence). IEEE Congress on. IEEE, 2008, pp. 1663-1670.

[38] M. N. Omidvar, X. Li, and X. Yao, "Cooperative co-evolution with delta grouping for large scale non-separable function optimization," in Evolutionary Computation (CEC), 2010 IEEE Congress on. IEEE, 2010, pp. 1-8. 
[39] S. Mahdavi, S. Rahnamayan, and M. E. Shiri, "Multilevel framework for large-scale global optimization," Soft Computing, pp. 1-30, 2016.

[40] M. N. Omidvar, X. Li, and X. Yao, "Smart use of computational resources based on contribution for cooperative co-evolutionary algorithms," in Proceedings of the 13th annual conference on Genetic and evolutionary computation. ACM, 2011, pp. 1115-1122.

[41] W. Chen, T. Weise, Z. Yang, and K. Tang, "Large-scale global optimization using cooperative coevolution with variable interaction learning," in Parallel Problem Solving from Nature, PPSN XI. Springer, 2010, pp. 300-309.

[42] M. Munetomo and D. E. Goldberg, "Linkage identification by nonmonotonicity detection for overlapping functions," Evolutionary computation, vol. 7, no. 4, pp. 377-398, 1999.

[43] L. Sun, S. Yoshida, X. Cheng, and Y. Liang, "A cooperative particle swarm optimizer with statistical variable interdependence learning," Information Sciences, vol. 186, no. 1, pp. 20-39, 2012.

[44] H. Ge, L. Sun, X. Yang, S. Yoshida, and Y. Liang, "Cooperative differential evolution with fast variable interdependence learning and cross-cluster mutation," Applied Soft Computing, vol. 36, pp. 300-314, 2015.

[45] M. Tezuka, M. Munetomo, and K. Akama, "Linkage identification by nonlinearity check for real-coded genetic algorithms," in Genetic and Evolutionary Computation-GECCO 2004. Springer, 2004, pp. 222 233.

[46] Y. Mei, M. N. Omidvar, X. Li, and X. Yao, "A competitive divideand-conquer algorithm for unconstrained large-scale black-box optimization," ACM Transactions on Mathematical Software (TOMS), vol. 42, no. 2, p. 13, 2016.

[47] M. N. Omidvar, M. Yang, Y. Mei, X. Li, and X. Yao, "DG2: A faster and more accurate differential grouping for large-scale black-box optimization," IEEE Transactions on Evolutionary Computation, 2017.

[48] X.-M. Hu, F.-L. He, W.-N. Chen, and J. Zhang, "Cooperation coevolution with fast interdependency identification for large scale optimization," Information Sciences, vol. 381, pp. 142-160, 2017.

[49] Z. Yang, K. Tang, and X. Yao, "Self-adaptive differential evolution with neighborhood search," in Evolutionary Computation, 2008. CEC 2008.(IEEE World Congress on Computational Intelligence). IEEE Congress on. IEEE, 2008, pp. 1110-1116.

[50] N. Hansen, "The CMA evolution strategy: A tutorial," Technique Report, 2011.

[51] D. Molina, M. Lozano, and F. Herrera, "MA-SW-Chains: Memetic algorithm based on local search chains for large scale continuous global optimization," in Evolutionary Computation (CEC), 2010 IEEE Congress on. IEEE, 2010, pp. 1-8.

[52] D. J. Sheskin, Handbook of parametric and nonparametric statistical procedures. CRC Press, 2003.

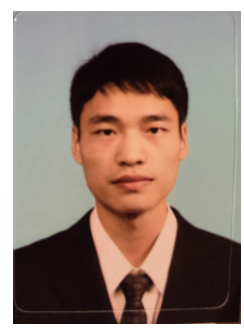

Yuan Sun received his B.Sc degree in theoretical and applied mechanics from Peking University, China, in 2013. He is currently working towards his Ph.D degree at the University of Melbourne, Australia. His doctoral work focuses on the analysis of interaction between decision variables in optimization and classification problems. His current research interests include exploratory landscape analysis, large scale optimization, multi-objective optimization, and feature selection.

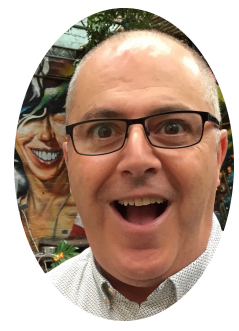

Michael Kirley received his B.Ed degree from Deakin University, Australia, in 1998, and his Ph.D degree from Charles Sturt University, Australia, in 2003. He is currently an Associate Professor at the Department of Computing and Information Systems at the University of Melbourne, Australia. His current research interests include theory and application of evolutionary computation, evolutionary game theory, multi-agent system, and complex systems science. He has published over 100 papers in these areas.

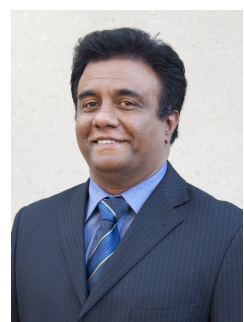

Saman K. Halgamuge received his B.Sc.Eng. degree in electronic and telecommunication engineering from the University of Moratuwa, Sri Lanka, and Dr.-Ing and Dipl.-Ing degrees in Electrical Engineering (Data Engineering) from TU Darmstadt, Germany in 1995 and 1990 respectively. He is a Professor and the Director, Research School of Engineering of Australian National University. He held appointments as Professor at the Department of Mechanical Engineering and Associate Dean International of Melbourne School of Engineering, University of Melbourne, Australia. He also holds the Professor V. K. Samaranayake Endowed visiting Chair at the University of Colombo, Sri Lanka. His research interests are data engineering, optimization, smart grids, mechatronics and bioinformatics. He has published over 250 papers and graduated $32 \mathrm{PhD}$ students in these areas and obtained substantial research grants from the ARC, NHMRS and industry. 